\title{
Design and Implementation of a Documentation Tool for Interactive Commandline Sessions
}

\author{
Andreas Dewald Felix C. Freiling Tim Weber \\ University of Mannheim \\ Technical Report TR-2010-005 \\ December 30, 2010
}

\begin{abstract}
In digital investigations it is important to document the examination of a computer system with as much detail as possible. Allthough never designed for digital investigations, many experts use the software script to record their whole terminal session while analyzing a target system. We analyze script's deficiencies and present the design and implementation of forscript (forensic script), a software providing additional capabilities and mechanisms for digital investigations.
\end{abstract}

\section{Introduction}

\subsection{Motivation: Documentation of Terminal Sessions}

During a digital investigation, many actions are still performed on the command line. Especially in live response or during complicated manual operations it is useful that the investigator keeps a detailed record of his actions while analyzing a system. Such a log can help the investigator understand his motivations in retrospect and can help prove to other experts that certain actions were or were not performed during the investigation.

In principle, interactive command line sessions can be documented quite easily by creating a piece of software that records everything typed on the keyboard and everything sent to the screen. Many digital investigators still use a program called script. The purpose of script is to record everything printed to the users terminal into a file. According to its manual, "[i]t is useful for students who need a hardcopy record of an interactive session as proof of an assignment" [1]. But is it suitable for digital investigations? 


\subsection{Motivation: Deficiencies of script}

The original script software consists of two programs: script and scriptreplay. Both are now part of the util-linux-ng package 8 , that is under active development. The most recent log entry within the source code of script and scriptreplay, however, dates back to July 2000 when Andreas Buer "added q-option".

Technically, script creates a new pseudo terminal (PTY), which is a virtual, software-based representation of a terminal line, and attach itself to the master side of it, thereby being able to send and receive data to and from an application connected to the slave side of the PTY. It launches a subprocess (also known as child), which launches the actual client application as its own subchild and then records the client applications output stream. The parent process forwards the users input to the client application. Recording terminates as soon as the child process exits.

Script uses a very simple file format to save the typescript. Everything the client application sends to the terminal, i.e., everything printed on screen, will be written to the file, byte by byte, including control characters that are used for various tasks like setting colors, positioning the cursor etc. Additionally, a header "Script started on $X \backslash \mathrm{n}$ " is written, where $X$ is the human-readable date and time when script was invoked. If script was invoked without the -q flag, an additional footer "Script done on $Y \backslash \mathrm{n}$ ", where $Y$ is the humanreadable date and time when script terminated, is written.

Apart from recording terminal in- and output, script can also record timing data: Using the option - $t$, script will output timing data to stderr specifying the chronological progress of the terminal session. Using this data, the utility scriptreplay can display the recorded data in a video-like way. The timing output format is very simple. It consists of tuples of delay and byte count (space-separated), one per line, like in the following example:

0.72516856

0.006549126

0.0400171

4.7279881

0.0479721

Each line can be read like $x$ seconds after the previous output, $n$ more bytes were sent to the terminal. If there was no previous output (because it is the first line of timing information), the delay specifies the time between script invocation and the first chunk of output.

The two file formats produced by script show several shortcomings with regard to their use in digital investigations:

- Input coming from the users keyboard is not logged at all. A common example is the user entering a command in the shell but then pressing ${ }^{\circ} \mathrm{C}$ instead of return. The shell will move to the next line and display the 
prompt again; there is no visible distinction whether the command was run or not 1

- Metadata about the environment script runs in is not logged. This leads to a high level of uncertainty when interpreting the resulting typescript, because even important information like the character set and encoding but also the terminal size and type is missing.

- Typescript and timing are separate files, but one logical entity. They should reside in one file to protect the user from confusion and mistakes.

- Appending to a typescript file is possible, but ambigious, since the beginning of a new part is determined only by the string "Script started on ". Also, appending to a typescript and recording timing information are incompatible, because scriptreplay will only ignore the first header line in a typescript file. Subsequent ones will disturb the timings byte counter.

- In a sense, script is a typical Unix utility written in a single C file with hardly any code documentation beyond the manual page. Therefore, it is rather cumbersome to read and requires some effort to understand. We believe that software used in digital investigations should strive for better readability such that it is easier to quickly convice an expert that the code actually does what it promised.

\subsection{Forensic script: forscript}

Overall, script has severe deficiencies when used in digital investigations. In this paper, we report on the design and implementation of a successor tool forscript ("forensic script"). The main advantages of this tool are:

- forscript has the same command line user interface as script, i.e., users used to script can seamlessly switch to forscript.

- forscript defines a portable and extensible file format that contains all user input, timing information and detailed information about the environment. The file format allows appending of files in a natural way.

- Following the paradigm of literate programming [4 and using the programming tool noweb [5], forscript comes with its own user manual and documentation. In fact, this version of the paper contains the entire $C$ source code in a well-readable way, i.e., this document is the program and vice versa.

${ }^{1}$ With more recent versions of Linux and Bash, terminals which have the ECHOCTL bit set (for example via stty) will show ${ }^{-} \mathrm{C}$ at the end of an interrupted line, which fixes this problem to some degree. Similar issues, like finding out whether the user entered or tab-completed some text, still persist. 
Literate programming [4] is a technique invented by Knuth when developing the $\mathrm{T}_{\mathrm{E}} \mathrm{X}$ typesetting system. Instead of writing more or less commented source code, it propagates writing a continuous text with embedded code fragments. The tool noweb [5] is used to reassemble these code fragments so that they can be compiled into an executable program. Because of its advantages in creating readable source code, we feel that there is much potential for the use of literate programming in the development of software for digital investigations.

\subsection{Roadmap}

In Section 2, we elaborate the user interface of forscript, which basically is that of script. In Section 3 we present the extensible file format of forscript. We give a brief insight into the implementation of forscript in Section 4 . The program is evaluated in Section 5 , giving an example transcript file. Finally, Section 6 summarizes the work, gives a description of forscripts limitations and describes possibilities of future work.

The appendix completes the code given in Section 4 to form a complete literate program and can be consulted at the discretion of the reader. A chunk and identifier index appear at the end of this document.

\section{User Interface of forscript}

Since Forscripts invocation syntax has been designed to be compatible to script, most parameters result in the same behavior. We now give an overview over the interface of forscript and highlight the differences to the interface of script.

Forscript takes one optional argument, the file name of the output file (also called transcript file) to which the terminal session is logged. If no file name was supplied on the command line, the default name is transcript. This differs from scripts default name typescript intentionally, because the file format is different and can, for example, not be displayed directly using cat. If there are any scripts or constructs that assume the default output file name to be typescript, the chance that replacing script with forscript will break their functionality anyway is quite high. If the file already exists and is a symbolic or hard link, forscript refuses to overwrite the file, as long as the file name is not explicitly provided.

There are several command-line switches that modify forscript's behavior. For example, using -a it is possible to append the output of forscript to a transcript file. If the target transcript file already exists and is non-empty, it has to start with a valid and supported file version header that will be explained below.

Normally, forscript displays a message when it starts or quits and also it records its startup and termination time in the typescript file. With the parameter -q, all of these messages will not appear (quiet). This is similar to the behavior of script, only that no startup message will be written to the 
transcript file. This is because scriptreplay unconditionally discards the first line in a typescript file and so writing the startup message ("Script started on ...") cannot be disabled in script.

By default, forscript will launch the shell specified by the environment variable \$SHELL. If \$SHELL it is not set, a default shell selected at compile time (/bin/sh, see page 32) is used. The shell will be called with $-i$ as its first parameter, making it an interactive shell. However, if forscript is called with the $-\mathrm{c}$ option followed by a command, it will launch the shell with -c and the command instead of $-i$. The shell will then be non-interactive and only run the specified command, then exit. Note that all POSIX-compatible shells have to support the $-i$ and $-c$ parameters. This behavior is identical to that of script.

If the $-f$ switch is used, forscript will call fflush() on the transcript file after new data has been written to it, resulting in instant updates to the typescript file, at the expense of performance. This behavior is identical to that of script and is useful for letting another user watch the actions recorded by forscript in real time.

The parameter - $t$ was used in script to output timing information. This parameter is accepted by forscript but ignored since forscript always records timing information into the transcript file.

Finally, if forscript is called with $-\mathrm{V}$ or --version as only parameter, it will print its version and exit. This behavior is identical to that of script.

If unsupported parameters are passed, forscript will print a short usage summary to stderr and exit. While running, the client applications output will be printed to stdout. Error messages will be printed to stderr.

\section{Forscript File Format}

This section explains the new file format as used by forscript. The file format allows an efficient combination of output and metadata within a single file.

A forscript data file (called a transcript file) consists of the mostly unaltered output stream of the client application, but includes blocks of additional data (called control chunks) at arbitrary positions. A control chunk is started by a shift out byte $(\mathrm{OxOe})$ and terminated by a shift in byte (0x0f). Each control chunk is either an input chunk or a metadata chunk.

\subsection{Input Chunks}

Input chunks contain the data that is sent to the client applications input stream, which is usually identical to the users keyboard input. They are of arbitrary length and terminate at the shift in byte. If a literal shift out or shift in byte needs to appear in an input chunks data, it is escaped by prepending a data link escape byte $(0 \times 10)$. If a literal data link escape byte needs to appear in an input chunks data, it has to be doubled (i.e., $0 \times 100 \times 10$ ). For example, if the user sends the byte sequence $0 \times 4$ e $0 \times 0 f \quad 0 \times 00$ 0x61 $0 \times 74$ 0x10, the complete 


\begin{tabular}{|l|l|l|}
\hline \hline binary value & type name & size \\
\hline $0 \times 01$ & file version & 1 byte \\
$0 \times 02$ & begin of session & 10 bytes \\
$0 \times 03$ & end of session & 1 byte \\
$0 \times 12$ & environment variables & arbitrary number of $\mathrm{C}$ strings \\
$0 \times 13$ & locale settings & $7 \mathrm{C}$ strings \\
$0 \times 16$ & delay & two 4-byte values \\
\hline
\end{tabular}

Table 1: forscript file format metadata chunk types.

input chunk written to the transcript file is $0 \times 0 e$ ex4e $0 \times 10$ ox0f $0 x 00$ ox61 0x74 0x10 0x10 0x0f.

\subsection{Metadata Chunks}

Metadata chunks, also called meta chunks, contain additional information about the file or the applications status, for example environment variables, terminal settings or time stamps. They contain an additional shift out byte at the beginning, followed by a byte that determines the type of metadata that follows. The available types are listed in Table 1 and implemented in Appendix A.3.

Meta chunks can be of arbitrary length and terminate at the shift in byte. The same escaping of shift out, shift in and data link escape that is used for input chunks is also used for meta chunks. For example, the terminal size meta type is introduced by its type byte $0 \times 11$, followed by width and heigth of the terminal, represented as two unsigned big-endian 16-bit integers. The information terminal size is 8016 characters would be written to the transcript file as $0 \times 0$ e $0 \times 0 e$ ox11 $0 \times 00 \quad 0 \times 50 \quad 0 \times 00 \quad 0 \times 10 \quad 0 \times 10 \quad 0 \times 0 f$. Note that the least significant byte of the number 16 has to be written as $0 \times 10 \quad 0 \times 10$ to prevent the special meaning of $0 \times 10$ to escape the following $0 \times 0 f$.

\subsection{Properties of the File Format}

This basic file format design has several advantages:

- New meta chunk types can be introduced while still allowing older tools to read the file, because the escaping rules are simple and the parsing application need not know a fixed length of each type.

- Since switching between input and output data occurs very often in a usual terminal session, the format is designed to require very little storage overhead for these operations.

- The format is very compact and easy to implement. Using a format like XML would decrease performance and require sophisticated libraries on the machine forscript is run on. However, for forensic usage it is best to be able to use a small statically linked executable. 
- Converting a forscript file to a script file is basically as easy as removing everything between shift out and shift in bytes (while respecting escaping rules, of course).

\section{Implementation Oveview}

We give a brief insight into the code of forscript that is assembled within a single $\mathrm{C}$ source file and written as a literate program [4] using the tool noweb [5]. The noweb tool automatically introduces cross-references between code chunks so that readers can quickly find the corresponsing code. We only show the most interesting code chunks here. The full details can be found in the appendix.

$\langle$ forscript.c $7 \mathrm{a}| \mathrm{a} \equiv$

$\langle$ declarations and definitions $15 \mathrm{~b}$

$\langle$ functions $15 \mathrm{c}\rangle$

$\langle$ main $7 \mathrm{~b}$

\subsection{The Main Program}

Here is the main() function. We first determine the program's name (as called on the command line), then we process the command line options. Afterwards we open the output file and a new pseudo terminal. The original script uses one process to listen for input, one to listen for output and one to initialize and execl() the command to be recorded. forscript in contrast uses only the select() function to be notified of pending input and output and therefore only needs two processes: itself and the subcommand. These two processes are forked after registering the appropriate signal handlers. Since neither the parent nor the child process should ever reach the end of main(), it returns EXIT_FAILURE.

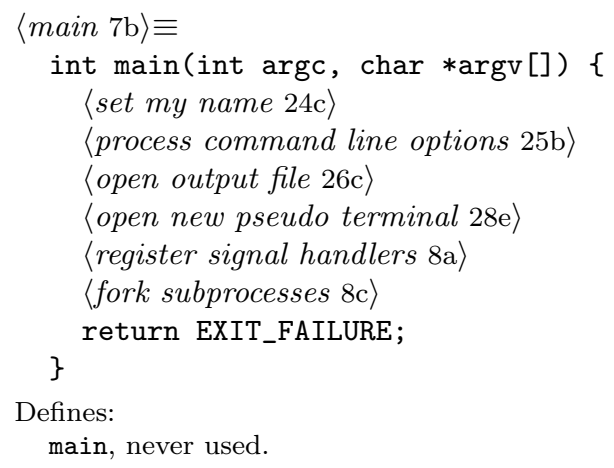

\subsection{Registering Signal Handlers}

To be notified of an exiting subprocess, a handler for the SIGCHLD signal needs to be defined. This signal is usually sent by the operating system if any child 
processs run status changes, i.e., it is stopped (SIGSTOP), continued (SIGCONT) or it exits. script terminates if the child is stopped, but forscript does not, because it uses the SA_NOCLDSTOP flag to specify that it wishes not to be notified about the child stopping or resuming. The function finish() handles the childs termination. The second signal handler, resized (), handles window size changes.

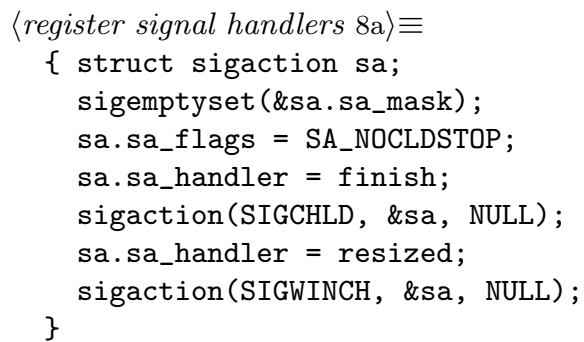

These functions and constants require signal.h.

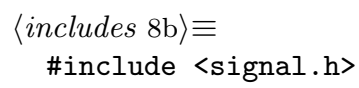

\subsection{Forking}

When a progam calls the fork() function, the operating system basically clones the program into a new process that is a subprocess of the caller. Both processes continue to run at the next command after the fork () call, but the value fork() returned will be different: The child will see a return value of 0 , while the parent will retrieve the process ID of the child. A negative value will be returned if the fork did not succeed.

8c Lfork subprocesses $8 \mathrm{c}$ 三

if $($ CHILD $=\operatorname{fork}())<0)\{$

perror ("fork");

die("fork failed", 0);

\}

Uses CHILD $8 \mathrm{~d}$ and die 23b

CHILD is used in several places when dealing with the subprocess, therefore it is a global variable.

8d $\langle$ globals $8 \mathrm{~d}$ 三

int CHILD = 0 ;

7b] 9

Defines:

CHILD, used in chunks $8 \mathrm{c}, 923 \mathrm{~b} 35$ and $37 \mathrm{~b}$ 
After forking, the child launches (or, to be exact, becomes) the process that should be logged within doshell, while the parent does the actual input/output logging within doio.

9 Lfork subprocesses $8 \mathrm{c}+\equiv$

if CHILD $==0$ )

doshell ();

else

doio ();

Uses CHILD $8 \mathrm{~d}$ doio $33 \mathrm{c}$ and doshell $31 \mathrm{c}$

Further code can be found in the appendix.

\section{Evaluation}

In order to show you what the code you have just seen actually does, this section contains instructions on how to compile it, and it features an example transcript file analyzed in detail.

\subsection{Compiling forscript}

forscript is written conforming to the C99 and POSIX-1.2001 standards, with portability in mind. It has been developed on a machine running Linux 2.6.32 [6], using glibc 2.10 and GCC 4.4.3 [2]. The following command line is an example of how to compile forscript:

gcc -std=c99 -Wl, -lrt -g -o forscript -Wall ।

-Wextra -pedantic -fstack-protector-all -pipe forscript.c

To generate forscript.c out of the noweb source code, the following command line can be used:

notangle -Rforscript.c forscript.nw > forscript.c

On the authors machine, forscript can be compiled without any compiler warnings. It has also been successfully compiled on NetBSD.

Since Apple Mac OS X in its current version 10.6.2 lacks support for the realtime extension of POSIX, the clock_gettime() function required by forscript is not natively available. Therefore the code described in this document can in its current state not be compiled on OS X. However, it should be possible to create a function emulating clock_gettime() and then port forscript to OS X.

\subsection{Example Transcript File}

To demonstrate forscripts output, the following pages contain a commented hex dump of a transcript file created on the authors machine. The dump has been created using hexdump $-\mathrm{C}$ transcript. Since metadata chunks do not 
necessarily start or end at a 16-byte border, the dump has been cut into distinct pieces, bytes not belonging to the current logical unit being replaced by whitespace. The hex dump consists of several three-colum lines. The first two columns contain 16 bytes of data represented in hexadecimal form, eight bytes each. The third column represents these 16 bytes interpreted as ASCII characters, nonprintable characters are replaced with a single dot.

The transcript starts with a file version chunk, specifying that version 1 is used:

Oe 0 e 0101 of

I....

Then a start of session chunk follows.

$003 c$ of

Oe 0 e 02 4b 82 d0 f3 04 4d 8 b e $3 \quad$ । .........।

Its first eight bytes, (4b to e3) tell you that the time is 1266864371.072190947 seconds after the epoch, which is February 22, 2010, 18:46:11 UTC. The next two bytes, $003 \mathrm{c}$ represent a timezone of 60 which translates to UTC+01:00.

After this chunk, the environment variables are listed. These are name=value pairs, separated by null bytes. This information is important to interpret the actual terminal data: For example, different control codes are used depending on the TERM variables setting.

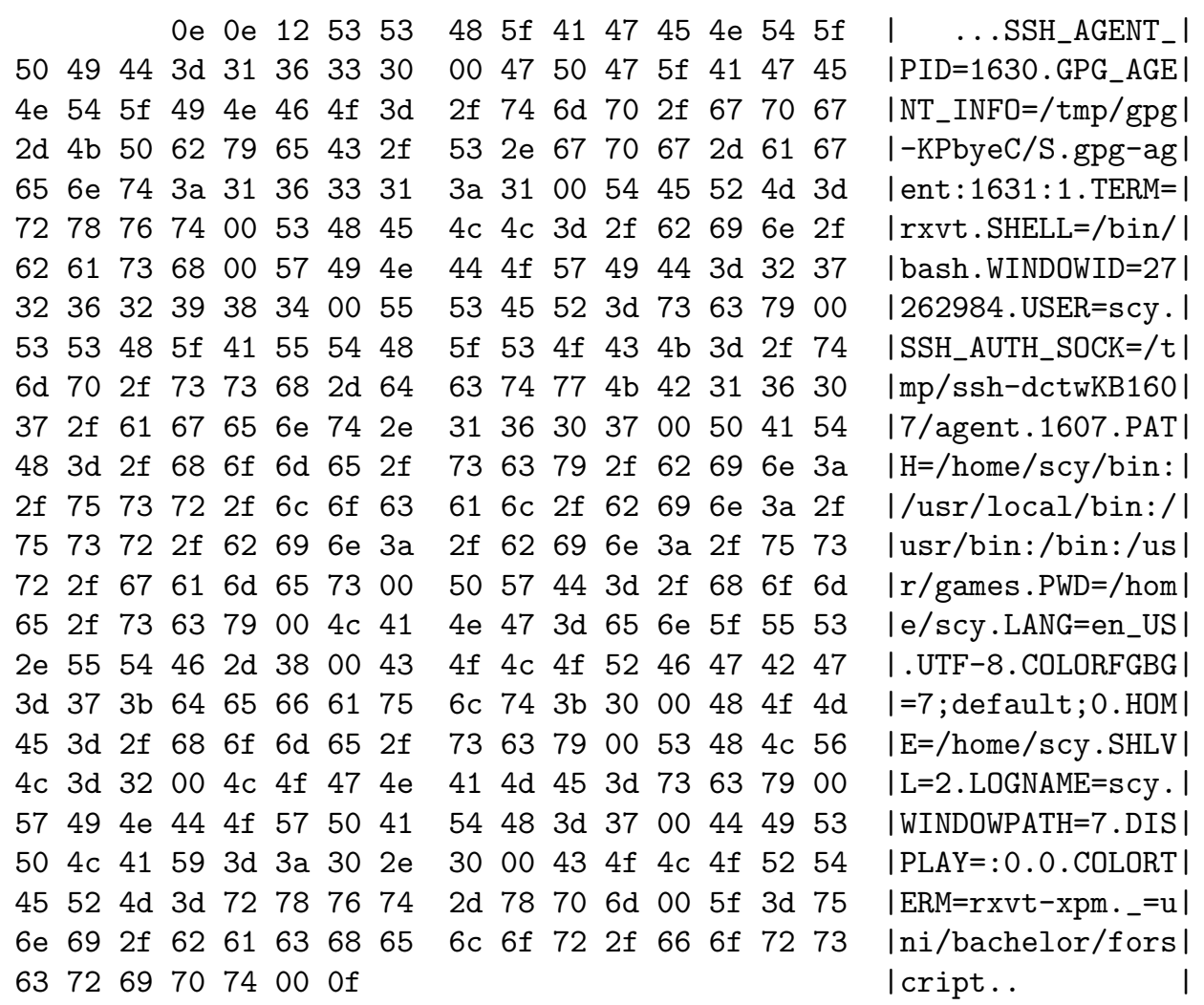


The next chunk contains the locale settings the C library uses for messages, number and currency formatting and other things. Although the user may choose different locales for either category, they are usually all the same. This example makes no difference: The system is configured for US English and a character encoding of UTF-8.

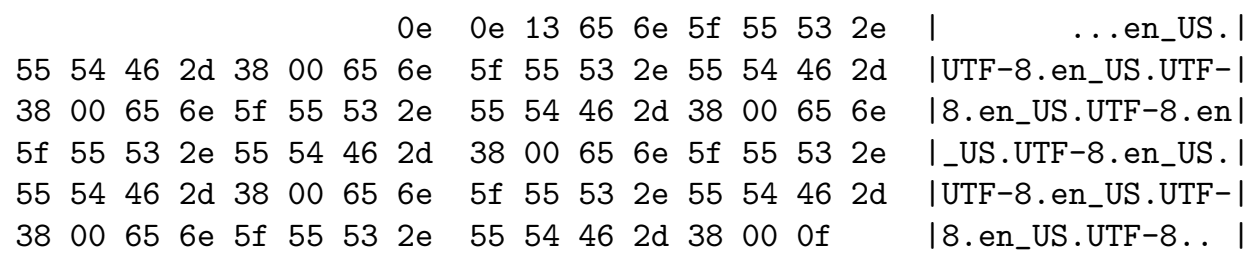

The terminal forscript is running in is 168 characters wide (00 a8) and 55 characters high (00 37), as the terminal size chunk shows:

0e 1100 a8 0037 of

oe $\mid \begin{aligned} & \mid \\ & \quad \mid \ldots 7 .\end{aligned}$

After all these metadata chunks, this is where actual terminal output starts. Since the -q flag was not used, forscript writes a startup message both to the terminal and the transcript, containing date and time and the file name. The final two bytes Od Oa represent the control codes carriage return and line feed. Note that in contrast to the Unix convention of using just line feed $(\backslash \mathrm{n})$ to designate new line in text files, a terminal (or at least the terminal the authors machine is using) requires both bytes to be present.

$\begin{array}{lllllllll}66 & 6 f & 72 & 73 & 63 & 72 & 69 & 70 & 74\end{array}$

$20 \quad 73 \quad 74 \quad 6172 \quad 74 \quad 65 \quad 64 \quad 20$ 6f 6 e 20 4d $6 f \quad 6 e$

$\begin{array}{llllllllllllllll}32 & 32 & 20 & 46 & 65 & 62 & 20 & 32 & 30 & 31 & 30 & 20 & 30 & 37 & 3 \mathrm{a} & 34\end{array}$

$\begin{array}{llllllllllllllll}36 & 3 \mathrm{a} & 31 & 31 & 20 & 50 & 4 \mathrm{~d} & 20 & 43 & 45 & 54 & 2 \mathrm{c} & 20 & 66 & 69 & 6 \mathrm{c}\end{array}$

$\begin{array}{llllllllllllllll}65 & 20 & 69 & 73 & 20 & 74 & 72 & 61 & 6 e & 73 & 63 & 72 & 69 & 70 & 74 & \text { od }\end{array}$ $0 \mathrm{a}$

Now the shell is started. It requires some time to read its configuration files and initialize the environment, therefore forscript has to wait for it and starts measuring the time until the next piece of data arrives. After the shell has initialized, it prints out its prompt. On this machine, the prompt (scy@bijaz master ? 0.11 19:46\$) is a rather complicated, colored one and therefore contains lots of ISO 6429 control codes (also known as ANSI escape codes) to define the visual appearance.

However, before the prompt is written to the data file, forscript writes a delay meta chunk: It took 0.065087679 seconds before the prompt was printed.

0e 0 e $1600 \quad 000000 \quad 03$ e1 28 bf of 16 bd $30 \quad$ | ...........

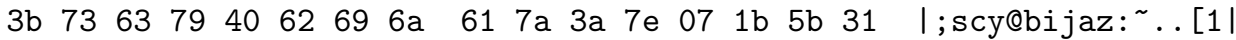

3b 3332 6d $73 \quad 63 \quad 79$ 1b 5 b 30 3b $33 \quad 32$ 6d 40 1b |;32mscy. [0;32m@.।

5b 31 3b $33 \quad 32$ 6d $62 \quad 69$ 6a 61 7a $1 \mathrm{~b}$ 5b 31 3b 33 |[1;32mbijaz.[1;3| 


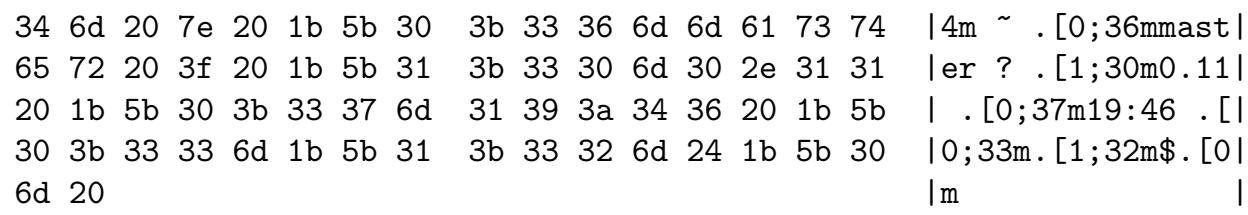

Next, 1.291995750 seconds after the prompt has been printed, the user types the letter e on the keyboard. The letter is enclosed by $0 e$ and $0 f$ in order to mark it as input data.

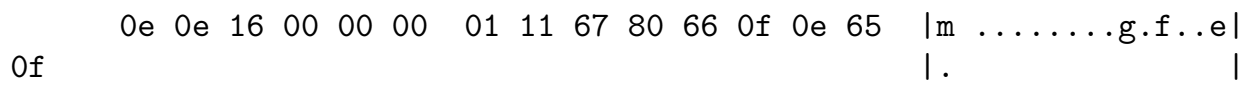

After the letter has been typed, the kernel will usually echo the character, that is, put it into the terminals output stream to make it appear on screen. It will take a small amount of time (in this case 0.0079911 seconds) until forscript receives the character and write it to the transcript file, this time declaring it as output.

0e 0 e $160000 \quad 00 \quad 00 \quad 0079$ ef $3 c$ of 65 | $\ldots \ldots . \mathrm{y} \cdot<. \mathrm{e} \quad \mid$

The user now continues to type the characters echo -1 , which will be echoed as well.

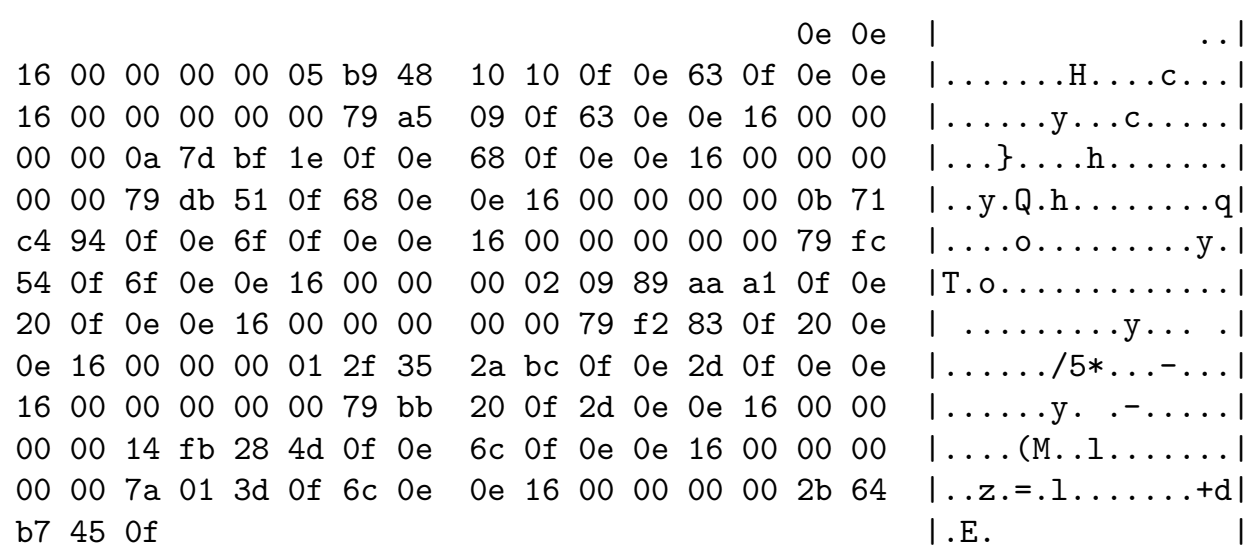

Since typing the 1 was a mistake, the user presses the backspace key (ASCII value 127) to remove the last character.

$$
\text { oe } 7 f \text { of । } \quad \text { । । }
$$

After the usual delay, the shell will send two things to the terminal: First, an ASCII backspace character (08) to position the cursor on the 1, then the ANSI code $C S I K$, represented by the bytes $1 \mathrm{~b} 5 \mathrm{~b} 4 \mathrm{~b}$, which will cause the terminal to make all characters at or right of the cursors position disappear.

$7 \mathrm{e}$ of $081 \mathrm{~b} 5 \mathrm{~b} 4 \mathrm{~b}$

0e 0 e 16000000000079 c2 l $\ldots \ldots .$. . I 
The user now enters the letter $\mathrm{n}$ and hits the return key (represented as ASCII byte 0d) in order to execute the command echo -n. After executing the command (which produces no output), the shell displays the prompt again.

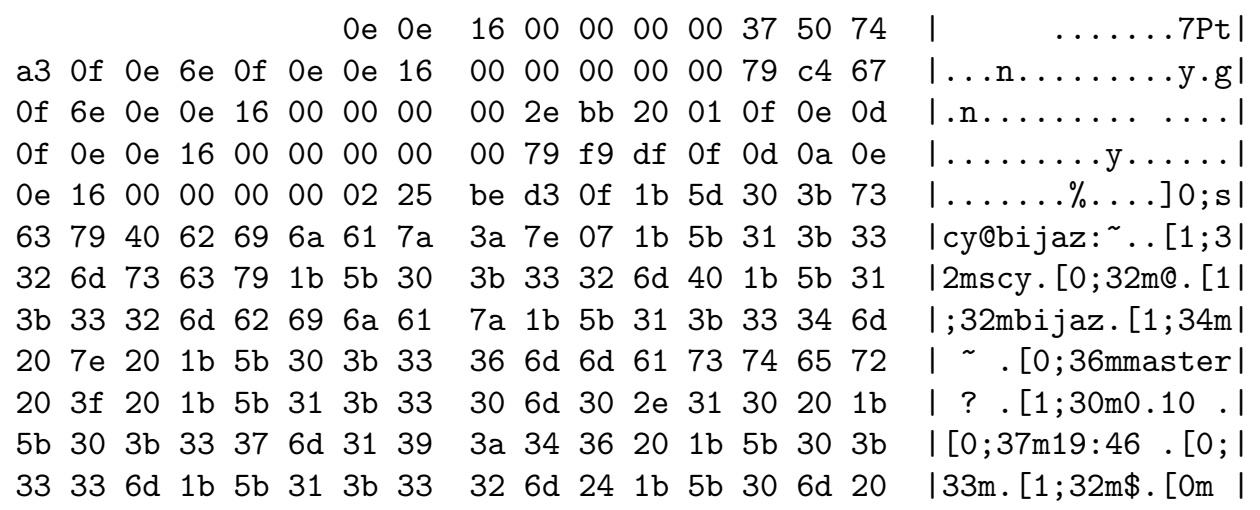

Note that without recording the users input, it would be impossible to determine whether the user pressed return to actually run the command or whether entering the command was cancelled, for example by pressing ${ }^{\mathrm{C}} \mathrm{C}$.

1.587984366 seconds later, the user decides to end the current session by pressing ` $D$, which is equivalent to the byte value 04 .

0e 0 e 160000000123 ob ed ee of 0 e 04 of $\quad \mid \ldots \ldots \ldots \ldots \ldots$

The shell reacts by printing exit and terminating. Then, forscript prints its shutdown message.

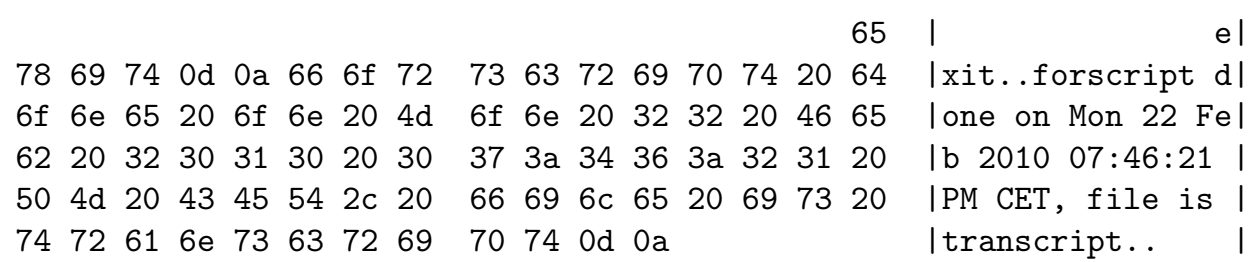

Finally, the exit status (0) of the shell is recorded in an end of session metadata chunk and the transcript file ends.

Of

0e 0 e $0300 \quad$ 1 $\quad \ldots .1$ |. $\mid$

\section{Conclusions and Future Work}

We presented why script, although often used for digital investigations, lacks features that are crucial for reliable documentation. A new software, forscript, has been designed and implemented, the weaknesses of script have been eliminated. 
The primary reason to develop forscript was the need to create a software that enables a forensic investigator to convert an interactive command-line session into a version suitable for inclusion in a printed report. While thinking about possible approaches, it became apparent that the output generated by script does not suffice to provide such a software with the information it needs to unambigously reconstruct what the user did. A tool that records the required information had to be developed first. Next, a tool that is able to parse the output forscript generates must be written. We leave this for future work.

forscript will be released by the third author as free software, available at a special website [7. Corrections and improvements are encouraged: forscript is far from being perfect and it is quite possible that during the development of additional tools, bugs and shortcomings will need to be fixed. Additionally, we will approach the maintainers of script and the forensic community as they can probably benefit from forscripts existence.

\section{References}

[1] BSD General Command Manual. Script(1). Manual page, part of util-linux$n g$ [8], July 2000.

[2] Free Software Foundation. Gnu compiler collection. http://gcc.gnu.org/, March 2010. release 4.4.3.

[3] Michael Kerrisk. The Linux man-pages project. http://www.kernel.org/ doc/man-pages/, 2010. release 3.23.

[4] Donald E. Knuth. Literate programming. The Computer Journal, 27(2):97111, 1984.

[5] Norman Ramsey. Literate programming simplified. IEEE Software, 11(5):97-105, 1994. The noweb system is available at http://www.cs. tufts.edu/ nr/noweb/.

[6] Linus Torvalds. The Linux kernel. http://www.kernel.org/, 2010. release 2.6.31.

[7] Tim Weber. forscript. http://scytale.name/proj/forscript/ release 1.0.0.

[8] Karel Zak. The util-linux-ng project. http://userweb.kernel.org/ kzak/ util-linux-ng/, 2010. current release 2.17. 


\section{A Implementation of forscript}

This section will describe the code of forscript in detail. You will learn how the software hooks into the input and output stream of the client application and how it reacts to things like window size changes or the child terminating. Other interesting topics include how to launch a subprocess and change its controlling terminal as well as how to read from multiple data streams at once without having to run separate processes.

\section{A.1 Overview}

Here's the current version number of forscript. Any future alterations can be explained in the text at relevant placed. MYVERSION is defined as a global constant.

$\langle$ globals $8 \mathrm{~d}$, $+\equiv$

const char $*$ MYVERSION $=" 1.0 .0 "$;

Defines:

MYVERSION, used in chunk 25b.

The code begins with feature test macros, ordinary macros and include statements. Afterwards, constants and global variables are defined.

$\langle$ declarations and definitions $15 \mathrm{~b} \equiv$

$\langle$ featuretest $27 \mathrm{~b}$.

$\langle$ macros $37 \mathrm{c}\rangle$

$\langle$ includes $8 \mathrm{~b}|$

$\langle$ constants $16 \mathrm{a}$.

$\langle$ globals 8d

15b $48 \mathrm{~d} 22 \mathrm{a}$

The functions used in the code are put in an order that makes sure every function is defined before it is called. Since die() is required at many places, it is put first. Next, all the chunk writing functions appear (the helper functions first). Then come the functions that write startup and shutdown messages on the screen, followed by the signal handling functions like finish. The functions doshell and doio are the main input/output functions that represent the parent and child processes.

$\langle$ chunkw 21

$\langle$ chunkwhf $22 \mathrm{~d}|$

$\langle$ chunkwm 23a

$\langle$ chunks 17 a

$\langle$ statusmsg 24b

$\langle$ done $37 \mathrm{~d}\rangle$

$\langle$ finish 37b

〈winsize $30 \mathrm{~b}$

$\langle$ resized 30a|

$\langle$ doshell $31 \mathrm{c}$

$\langle$ doio 33c 


\section{A.2 Constants}

For improved readability, we define the special characters introduced in the previous section as constants:

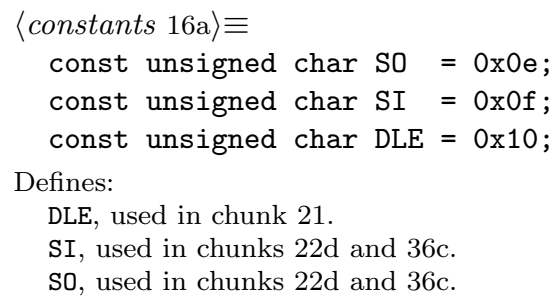

It is by design that the three special characters have consecutive byte numbers. This allows us to define a minimum and maximum byte value that requires special escape handling:

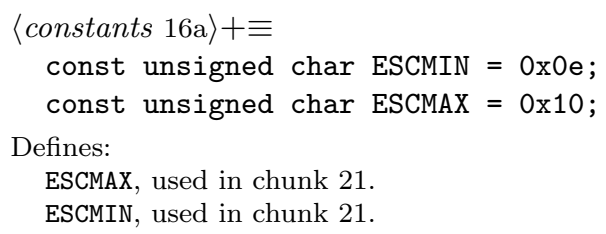

\section{A.3 Metadata Chunk Types}

We now describe the available metadata chunk types. Integers are unsigned and big endian, except where noted otherwise. In the resulting file, numbers are represented in binary form, not as ASCII digits.

For better understanding, the code forscript uses to write each meta chunk appears after the chunks explanation. The three functions chunkwh(), chunkwf() and chunkwd() that are used for actually writing the data to disk will be explained in section A.5. To be able to understand the code, it is sufficient to know that chunkwh () takes one parameter (the chunks type) and writes the header bytes. chunkwf() writes the footer byte and takes no parameters, while chunkwd() writes the payload data, escaping it on the fly, and requires a pointer and byte count. There is an additional convenience function chunkwm() that takes all three parameters and will write a complete metadata chunk.

All chunk functions return a negative value if an error occured, for example if an environment setting could not be retrieved or if writing to the transcript file failed. Since only a partial metadata chunk may have been written to the transcript, the file is no longer in a consistent state. Therefore, forscript should terminate whenever a chunk function returns a negative value.

A transcript file needs to begin with a file version meta chunk, followed directly by the first start of session chunk. 


\section{0x01 File Version (1 byte)}

The transcript file must start with a meta chunk of this type; there may be no other data before it.

Denotes the version of the forscript file format that is being used for this file. In order to guarantee a length of exactly one byte, the version numbers 0 , 14, 15 and 16 are not allowed, therefore no escaping takes place. This document describes version 1 of the format, therefore currently the only valid value is $0 \times 01$.

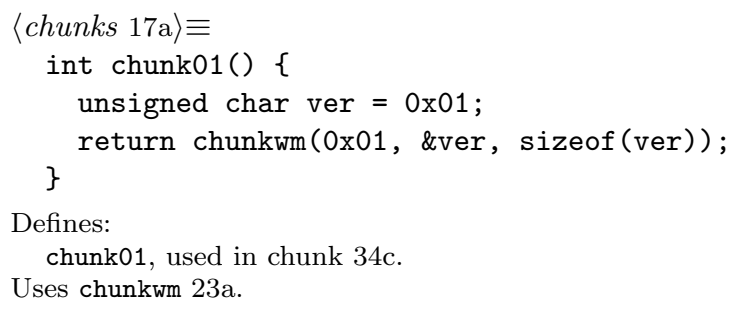

\section{0x02 Begin of Session (10 bytes)}

Denotes the start of a new forscript session. The first four data bytes represent the start time as the number of seconds since the Unix Epoch. The next four bytes contain a signed representation of the nanosecond offset to the number of seconds. If these four bytes are set to 0xffffffff, there was an error retrieving the nanoseconds. The last two bytes specify the machines (signed) time zone offset to UTC in minutes. If these two bytes are set to Oxffff, the machines timezone is unknown.

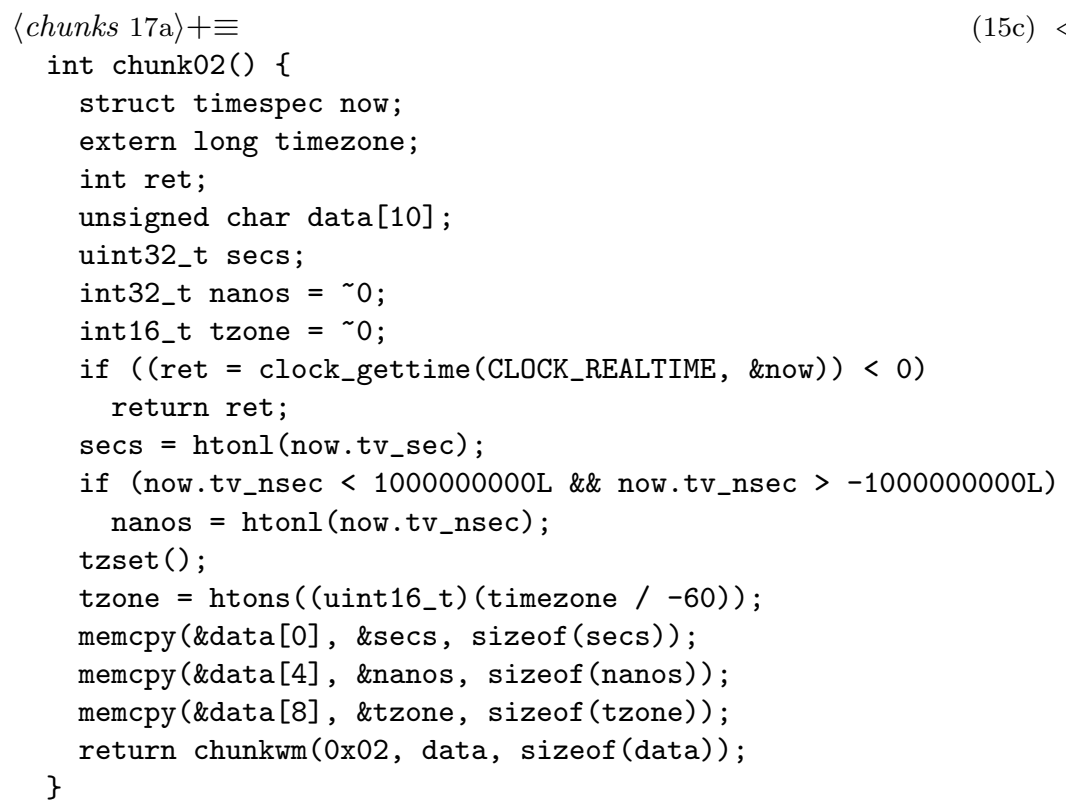


Defines:

chunk02, used in chunk $34 \mathrm{c}$

Uses chunkwm 23a

This chunk requires the headers time.h for clock_gettime(), inet.h for htonl() and string.h for memcpy():

18a $\langle$ includes $8 \mathrm{~b}+\equiv$

\#include <time.h>

\#include <arpa/inet.h>

\#include <string.h>

\section{0x03 End of Session (1 byte)}

Denotes the end of a forscript session. The data byte contains the return value of the child process. The usual exit code convention applies: If the child exited normally, use its return value. If the child was terminated as a result of a signal (like SIGSEGV), use the number of the signal plus 128 .

The parameter status should contain the raw status value returned by wait(), not only the childs return value. If the exit code of the child could not be determined, Oxff is used instead.

$\langle$ chunks $17 \mathrm{a}+\equiv$

int chunk03(int status) \{

unsigned char data $=\sim 0$;

if (WIFEXITED(status))

data $=$ WEXITSTATUS (status);

else if (WIFSIGNALED (status))

data $=128+$ WTERMSIG (status);

\}

return chunkwm(0x03, \&data, sizeof (data));

Defines:

chunk03, used in chunk 38d

Uses chunkwm 23a

\section{0x11 Terminal Size (two 2-byte values)}

Is written at session start and when the size of the terminal window changes. The first data word contains the number of colums, the second one the number of rows.

Since the terminal size has to be passed to the running client application, the chunk itself does not request the values, but receives them as a parameter.

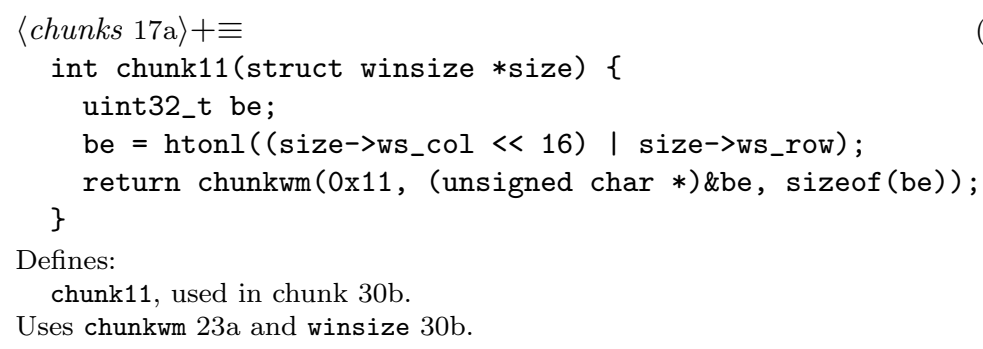




\section{0x12 Environment Variables (arbitrary number of C strings)}

Is written at session start. Contains the environment variables and their values as $\mathrm{NAME}=$ value pairs, each pair is terminated by a null byte $(0 \times 00)$. Since variable names may not contain the $=$ character and neither variables names nor the values may include a null byte, the list needs no special escaping.

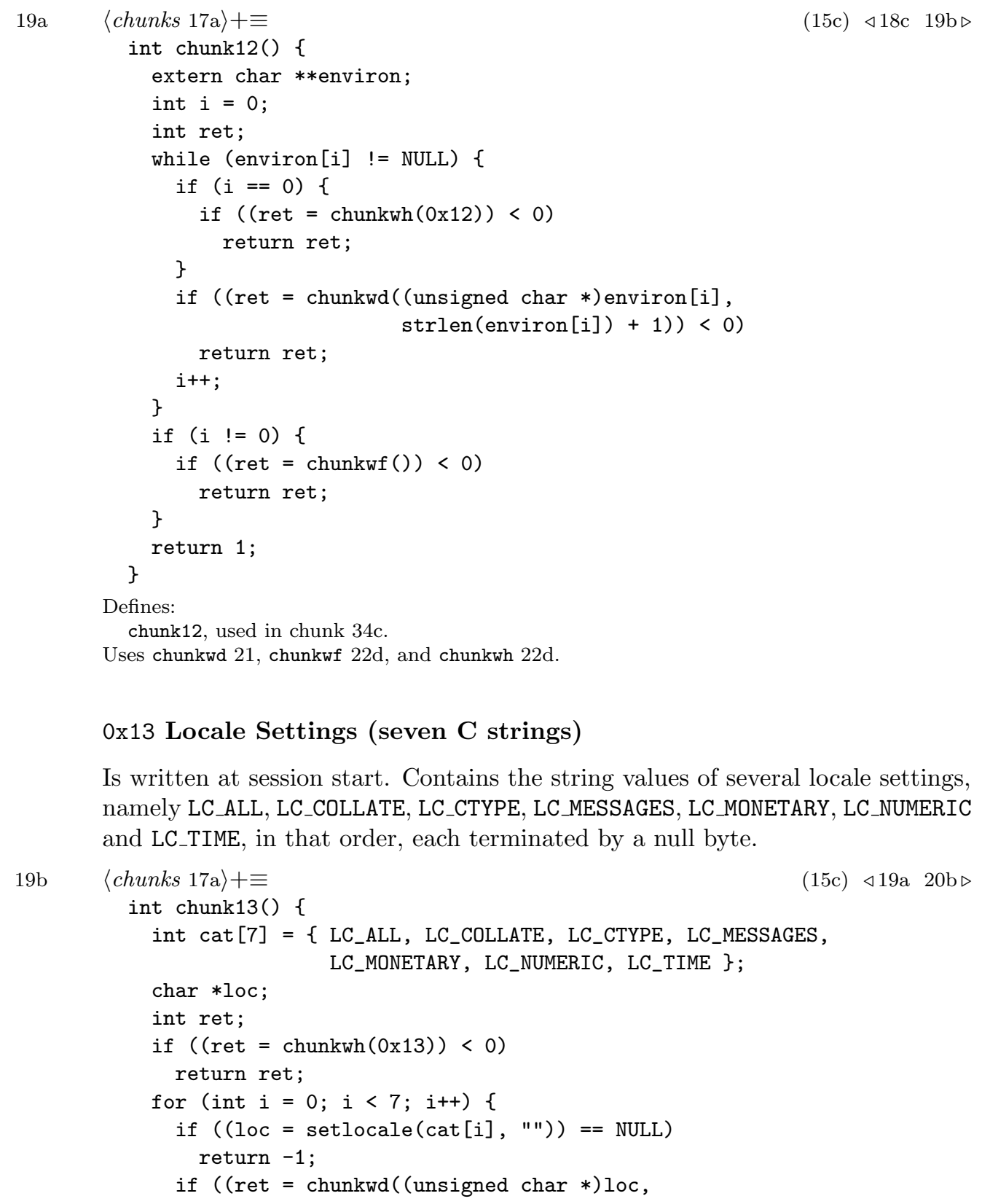

0x13 Locale Settings (seven C strings)

Is written at session start. Contains the string values of several locale settings, namely LC_ALL, LC_COLLATE, LC_CTYPE, LC_MESSAGES, LC_MONETARY, LC_NUMERIC and LC_TIME, in that order, each terminated by a null byte. 


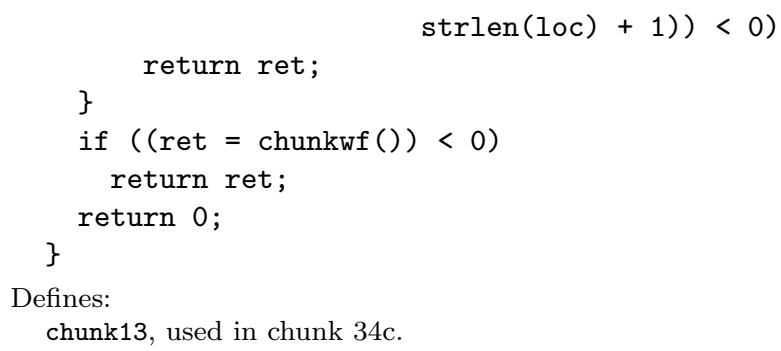

\section{0x16 Delay (two 4-byte values)}

Contains the number of seconds and nanoseconds that have passed since the last delay chunk (or, if this is the first one, since the session started).

A replaying application should wait for the time specified in this chunk before advancing further in the transcript file.

Since the seconds and nanoseconds are represented as integers, converting to a floating-point number would mean a loss of precision. Therefore both integers are subtracted independently. If the nanoseconds part of now is less than that of $t s$, the seconds part has to be decreased by one for the result to be correct.

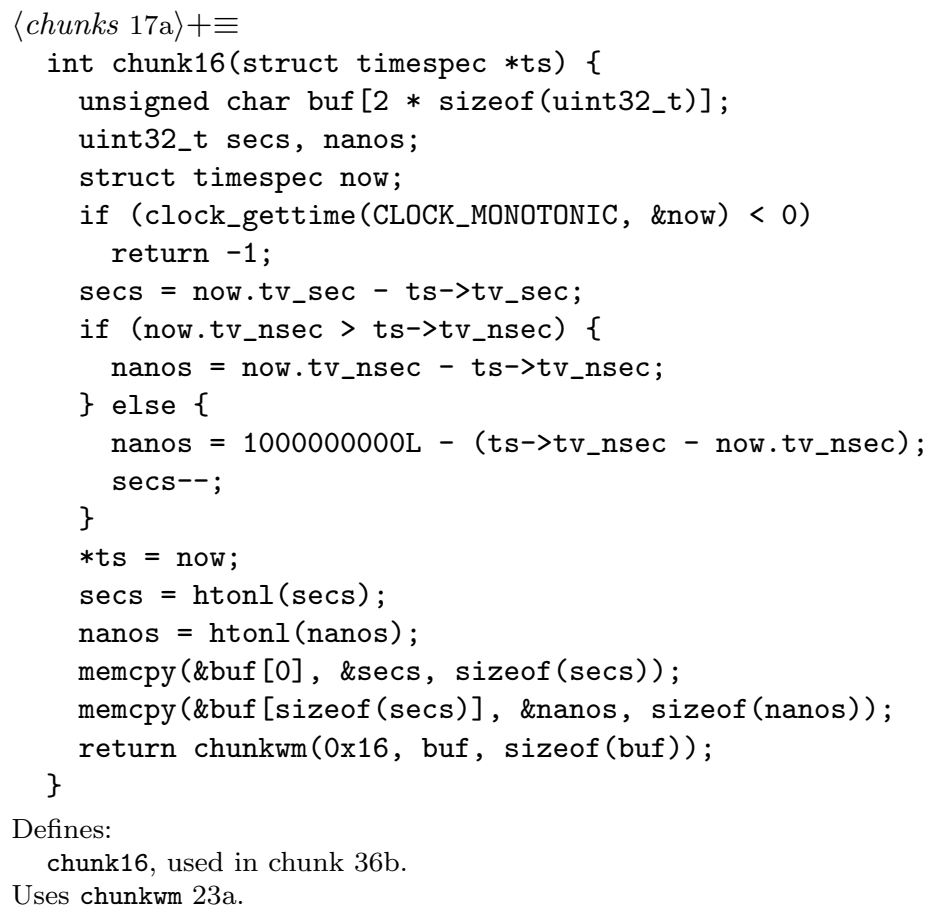




\section{A.4 Magic Number}

Since a forscript file has to start with a file version chunk followed by a begin of session chunk, there is a distinctive eight-byte signature at the beginning of each file:

Ox0e 0x0e 0x01 0x?? OxOf 0x0e 0x0e 0x02

The first two bytes start a metadata chunk, the third one identifies it as a file version chunk. The fourth byte contains the version number, which is currently 0x01 but may change in the future. Byte 5 closes the version chunk, 5 to 8 start a begin of session chunk.

\section{A.5 Writing Metadata Chunks to Disk}

The function chunkwd() takes a pointer and a byte count as arguments and writes chunk data to the transcript file, applying required escapes on the fly. To improve performance, it does not write byte-by-byte, but instead scans the input data until it finds a special character. When it does, it writes everything up to, but not including, the special character to the file and then adds a DLE character. The search then goes on. If another special character is found, everything from the last special character (inclusive) to the current one (exclusive) plus a DLE is written. Eventually the whole input data will have been scanned and the function terminates after writing everything from the last special character (inclusive) or the beginning of the data (if there were no special characters) to the end of the input data. This is the code:

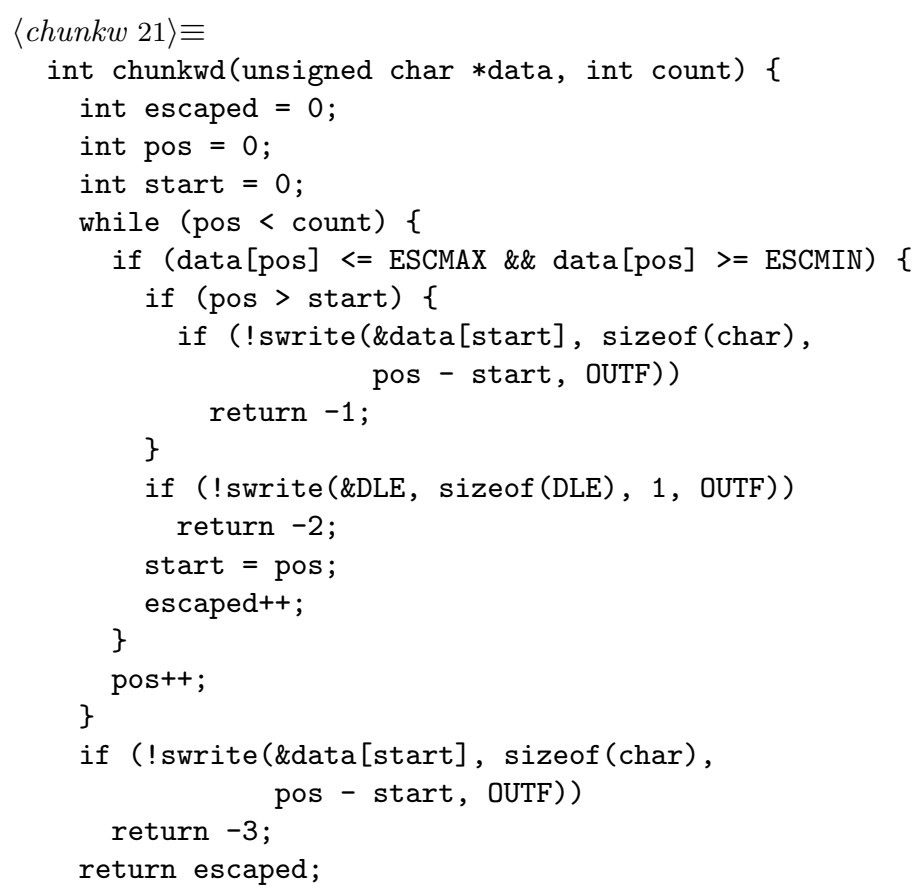


Defines:

chunkwd, used in chunks $19,23 \mathrm{a}$ and $36 \mathrm{c}$

Uses DLE $16 \mathrm{a}$ ESCMAX $16 \mathrm{~b}$ ESCMIN $16 \mathrm{~b}$, OUTF $22 \mathrm{a}$ and swrite $22 \mathrm{~b}$

OUTF is the already opened transcript file and a global variable:

$22 \mathrm{a}$

$\langle$ globals $8 \mathrm{~d}$ - $+\equiv$

FILE *OUTF;

15b) $4 \mathrm{a} 24 \mathrm{a}$

Defines:

OUTF, used in chunks 21, 22d 24b 27, 31c and 36-38

The swrite () function (safe write) that is being used here will return zero if the number of items written is not equal to the number of items that should have been written:

$22 \mathrm{~b}\langle$ swrite $22 \mathrm{~b}\rangle$

int swrite (const void *ptr, size_t size, size_t nmemb, FILE *stream) \{

\}

return (fwrite(ptr, size, nmemb, stream) == nmemb);

Defines:

swrite, used in chunks 21 and $22 \mathrm{~d}$

To be able to use fwrite(), stdio.h has to be included:

22c <includes $8 \mathrm{~b}+\equiv$

\#include <stdio.h>

15b $420 \mathrm{a} 23 \mathrm{c}>$

There are functions to write chunk headers and footers:

22d <chunkwhf $22 \mathrm{~d}$ | $\equiv$

int chunkwh(unsigned char id) \{

int ret;

for (int $i=0 ; i<2 ; i++)\{$

ret $=$ swrite $(\& \mathrm{SO}$, sizeof $(\mathrm{SO}), 1$, DUTF $)$;

if (!ret)

return -1 ;

\}

return swrite(\&id, sizeof (unsigned char),

\}

1 , OUTF ) ? $1:-1$;

int chunkwf() \{

\}

return (swrite $(\& \mathrm{SI}$, sizeof (SI), 1, OUTF ) ? $1:-1$;

Defines:

chunkwf, used in chunks 19 and 23a

chunkwh, used in chunks 19 and $23 \mathrm{a}$

Uses OUTF 22a sI 16a so 16a and swrite 22b 
There is also a convenience function that writes a meta chunks header and footer as well as the actual data:

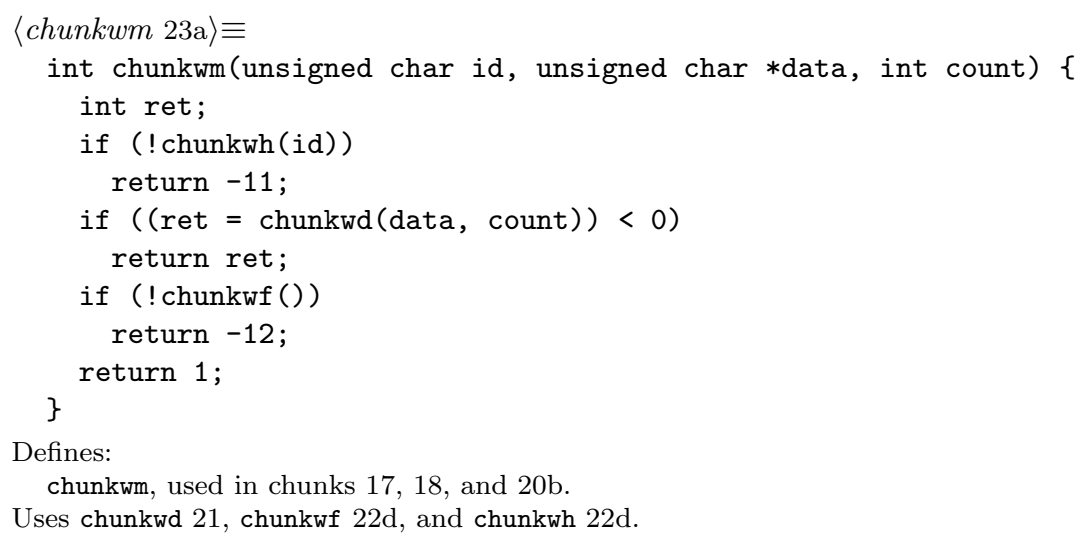

\section{A.6 Error Handling}

If the program has to terminate abnormally, the function die() will be called. After resetting the terminal attributes and telling a possible child process to exit, it will output an error message and exit the software.

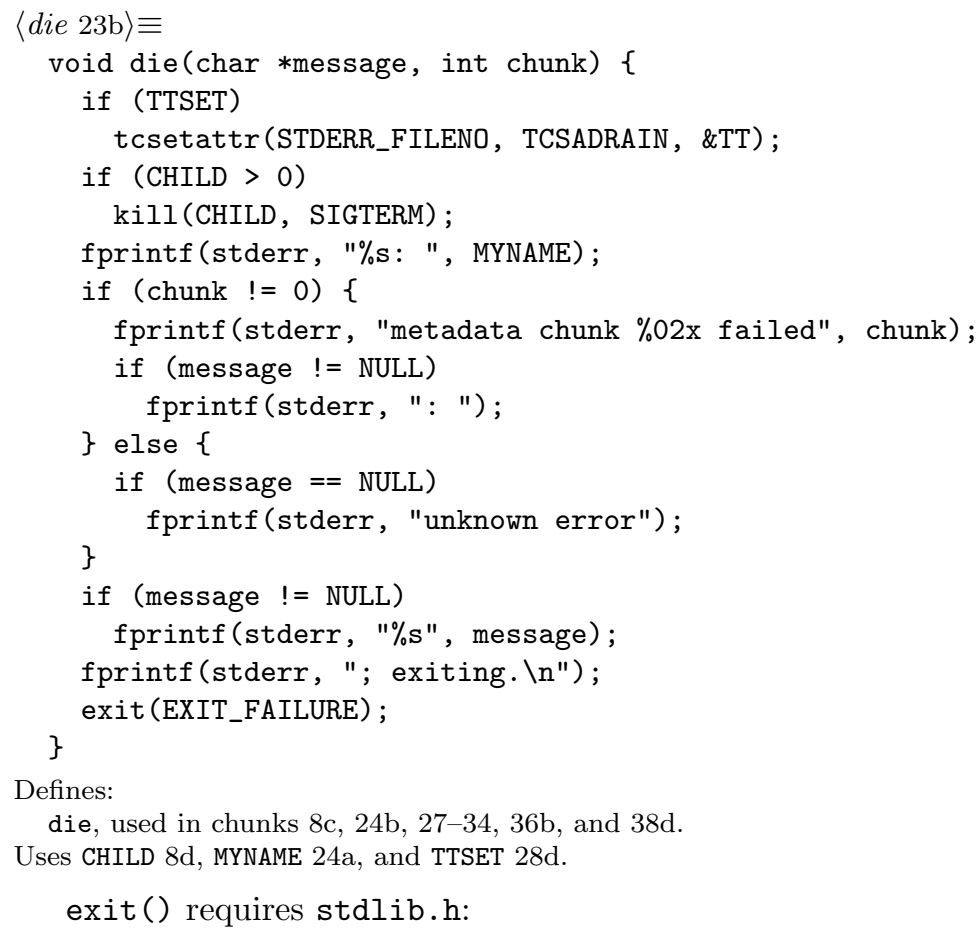


The global variable MYNAME contains a pointer to the name the binary was called as and is set in main().

24a $\langle$ globals $8 \mathrm{~d}\rangle+\equiv$

char * MYNAME;

15b) $\triangleleft 22 \mathrm{a} 25 \mathrm{~d} p$

Defines:

MYNAME, used in chunks $23-26$

\section{A.7 Startup and Shutdown Messages}

The statusmsg() function writes a string to both the terminal and the transcript:

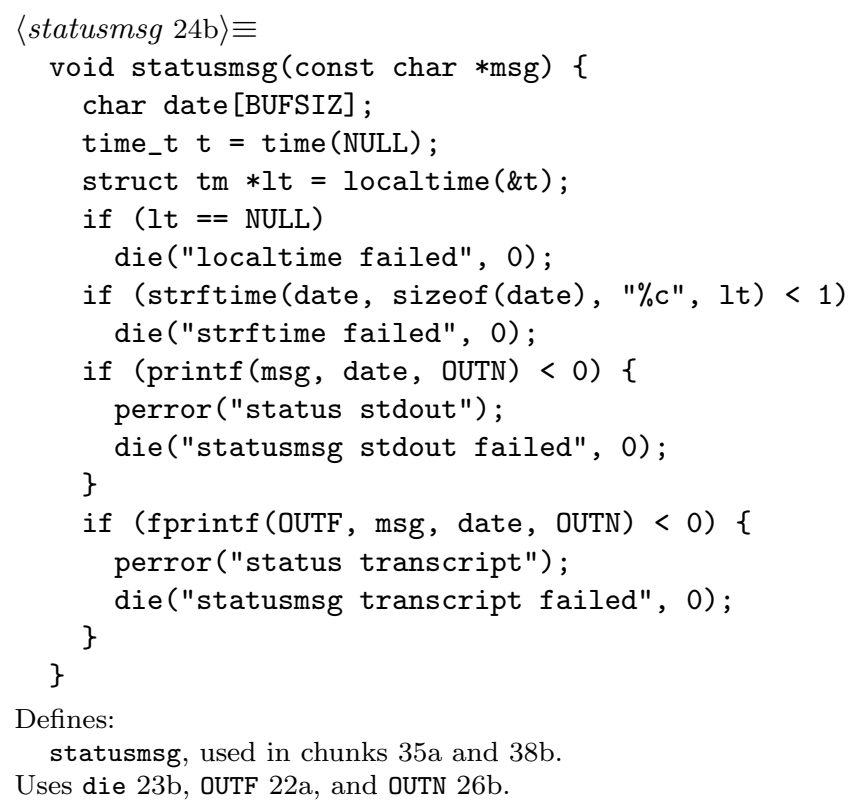

\section{A.8 Initialization}

\section{A.8.1 Determining the Binarys Name}

To be able to output its own name (e.g. in error messages), forscript determines the name of the binary that has been called by the user. This value is stored in argv [0]. The global variable MYNAME will be used to reference that value from every function that needs it.

24c <set my name 24c $\equiv$ MYNAME $=\operatorname{argv}[0]$;

7b 25a

Uses MYNAME 24a 
If forscript was called using a path name (e.g. /usr/bin/forscript), everything up to the final slash needs to be cut off. This is done by moving the pointer to the character immediately following the final slash.

25a <set my name $24 \mathrm{c}+\equiv$

$\{$ char *lastslash;

7b $424 \mathrm{c}$

if $(($ lastslash $=\operatorname{strrchr}$ (MYNAME, ' $/$ ') $) \quad !=$ NULL)

\}

MYNAME $=$ lastslash +1 ;

Uses MYNAME 24a

\section{A.8.2 Processing Command Line Arguments}

Since forscripts invocation tries to mimic scripts as far as possible, command line argument handling is designed to closely resemble scripts behavior. Therefore, like in script, the command line switches --version and $-\mathrm{V}$ are treated separately. If there is exactly one command line argument and it is one of these, forscript will print its version and terminate.

$25 \mathrm{~b}\langle$ process command line options $25 \mathrm{~b} \equiv$ if $((\operatorname{argc}==2)$ \&\&

$(! \operatorname{strcmp}(\operatorname{argv}[1],, "-V ")|| \quad ! \operatorname{strcmp}(\operatorname{argv}[1]$, "--version"))) \{

printf ( $1 \% \mathrm{~s} \% \mathrm{~s} \backslash \mathrm{n} "$, MYNAME, MYVERSION) ;

return 0

Uses MYNAME 24a and MYVERSION 15a

The other options are parsed using the normal getopt() method, which requires unistd.h.

$\langle$ includes $8 \mathrm{~b}+\equiv$

\#include <unistd.h>

$15 \mathrm{~b} \triangleleft 23 \mathrm{c} 2 \mathrm{2a}$

getopt () returns the next option character each time it is called, and -1 if there are none left. The option characters are handled in a switch statement. As in script, flags that turn on some behavior cause a respective global int variable to be increased by one. These flags are:

25d 〈globals $8 \mathrm{~d}+\equiv$

int $a f l g=0, f f l g=0, q f l g=0$;

15b) $424 \mathrm{a} 25 \mathrm{e}>$

Defines:

aflg, used in chunks 26, 27 and $34 \mathrm{c}$

The value of the $-c$ parameter is stored in a global string pointer:

$25 \mathrm{e}\langle$ globals $8 \mathrm{~d}+\equiv$

char *cflg = NULL;

15b $\triangleleft 25 \mathrm{~d} 26 \mathrm{~b}>$

Defines:

cflg, used in chunks $26 \mathrm{a}$ and $32 \mathrm{~d}$ 
The - $t$ flag is accepted for compatibility reasons, but has no effect in forscript because timing information is always written.

After the loop terminates, optind arguments have been parsed. argc and argv are then modified accordingly to only handle non-option arguments (in forscript this is only the file name).

The parsing loop therefore looks like this:

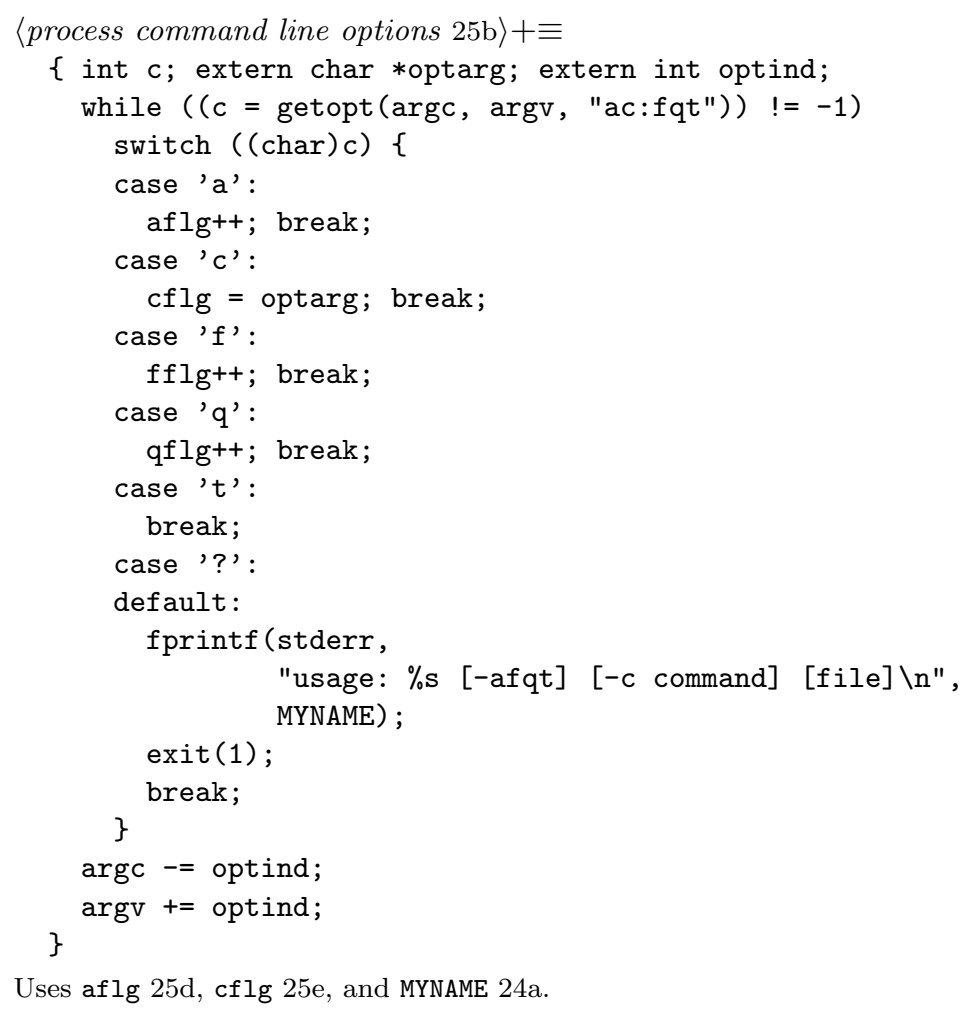

After the options have been parsed, the output file name will be determined and stored in the global string OUTN

\section{A.8.3 Opening the Output File}

As in script, there is a safety warning if no file name was supplied and transcript exists and is a (hard or soft) link.

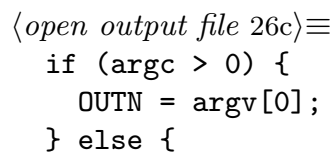




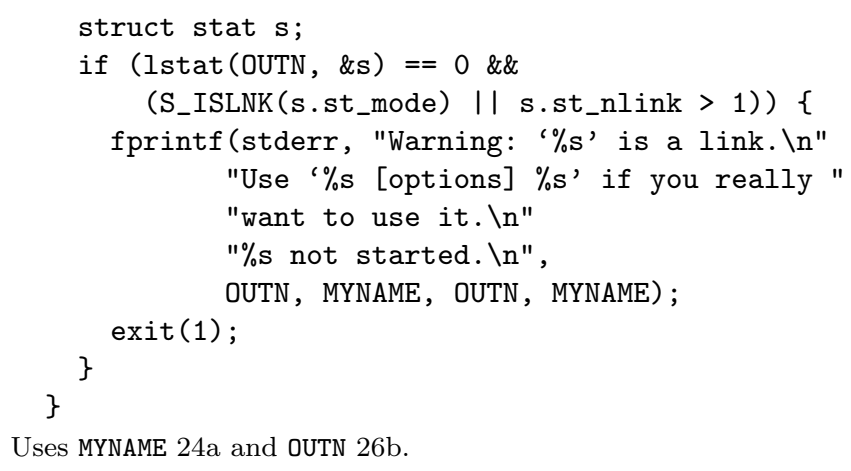

lstat() needs types.h and stat.h as well as _XOPEN_SOURCE:

$27 \mathrm{~b}\langle$ featuretest $27 \mathrm{~b} \equiv$

\#define _XOPEN_SOURCE 500

15b) $\triangleleft 25 \mathrm{c} 28 \mathrm{a}>$

Defines:

XOPEN_SOURCE, never used.

The file will now be opened, either for writing or for appending, depending on aflg. Note that if appending, the file will be opened for reading as well. This is because forscript checks the file version header before appending to a file.

〈open output file $26 \mathrm{c}+\equiv$

Uses aflg 25d die 23b OUTF 22a, and OUTN 26b

If the file has been opened for appending, check whether it starts with a compatible file format. Currently, the only format allowed is $0 x 01$. If the file is empty, appending is possible, but the file version chunk has to be written. This is done by setting aflg to 0, which will cause doio() to write the chunk.

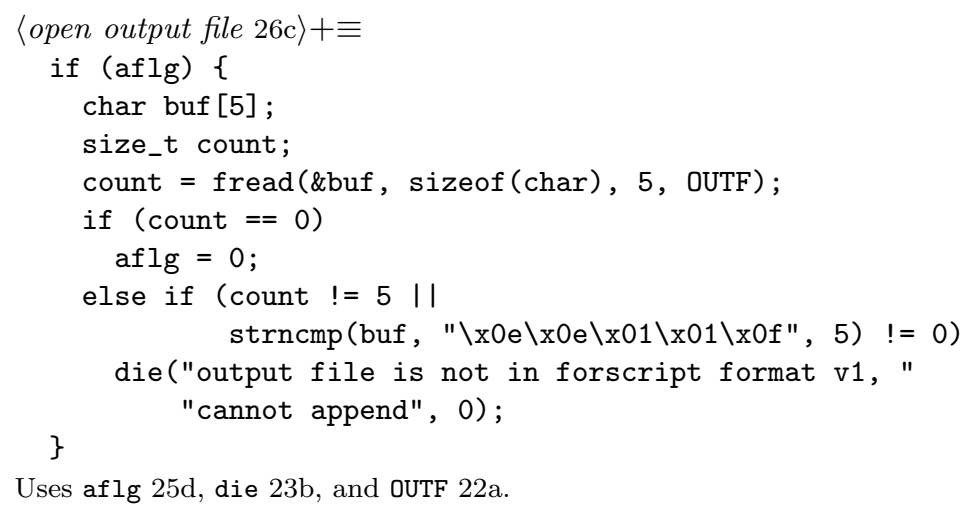




\section{A.9 Preparing a New Pseudo Terminal}

While script uses manual PTY allocation (by trying out device names) or BSDs openpty() where available, forscript has been designed to use the Unix 98 PTY multiplexer (/dev/ptmx) standardized in POSIX.1-2001 to create a new PTY. This method requires $f(n t l . h$ and a sufficiently high feature test macro value for POSIX code.

Defines:

POSIX_C_SOURCE, never used.

The PTYs master and slave file descriptors will be stored in these global variables:

Defines:

PTM, used in chunks 29-31 34-36 and 38d

Additionally, the settings of the terminal forscript runs in will be saved in the global variable TT. This variable is used to duplicate the terminals settings to the newly created PTY as well as to restore the terminal settings as soon as forscript terminates. There is also a variable TTSET which stores whether the settings have been written to TT. This is important when restoring the terminal settings after a failure: If the settings have not yet been written to TT, applying them will lead to undefined behavior.

TTSET, used in chunks 23b and $28 \mathrm{e}$

The termios structure is defined in termios.h.

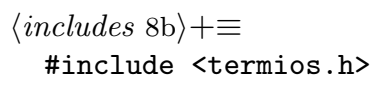


A new PTY master is requested like this:

29a Lopen new pseudo terminal 28e $+\equiv$

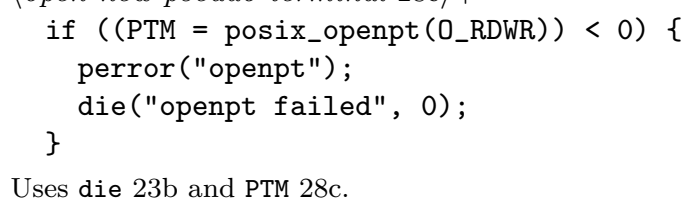

Uses die 23b and PTM 28c

Then, access to the slave is granted.

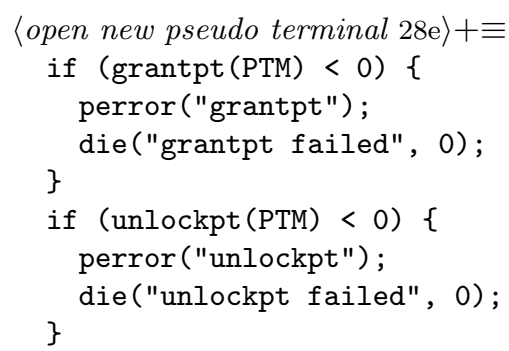

Uses die 23b and PTM 28c

The slaves device file name is requested using ptsname(). Since the name is not needed during further execution, the slave will be opened and its file descriptor stored.

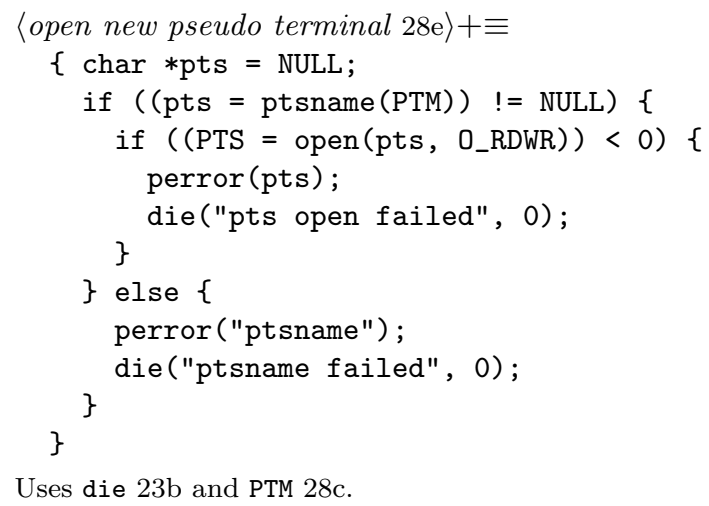

The parent terminal will be configured into a raw mode of operation. script does this by calling cfmakeraw(), which is a nonstandard BSD function. For portability reasons forscript sets the corresponding bits manually, thereby emulating cfmakeraw(). The list of settings is taken from the termios(3) Linux man page [3] and should be equivalent. Afterwards, the settings of the terminal forscript was started in will be copied to the new terminal. This means that in the eyes of the user the terminals behavior will not change, but forscript can now document the terminals data stream with maximum accuracy.

(7b) 429 


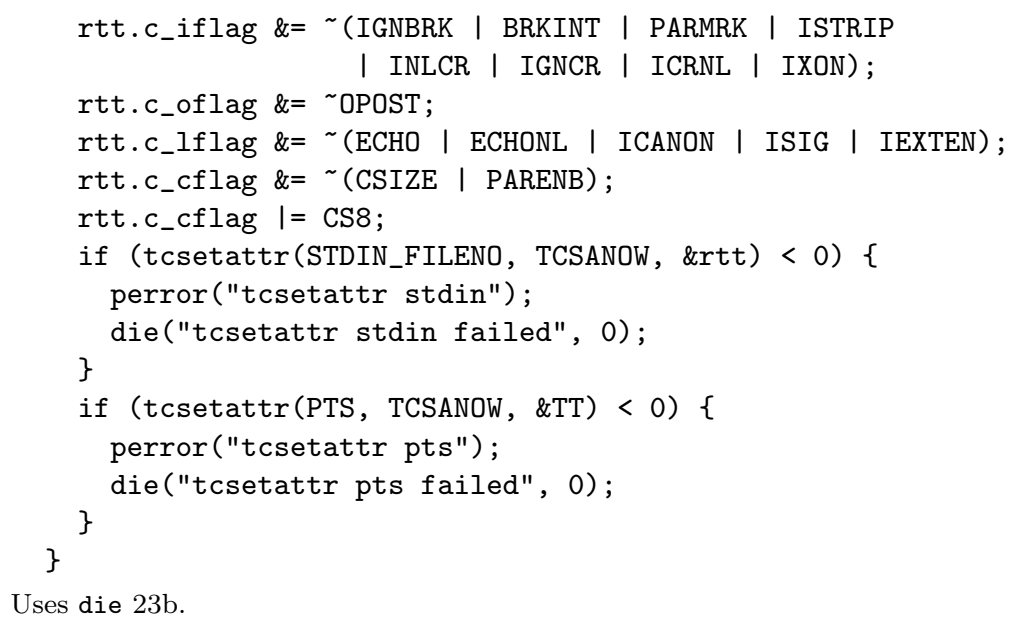

\section{A.9.1 Managing Window Size}

If the size of a terminal window changes, the controlling process receives a SIGWINCH signal and should act accordingly. forscript handles this signal in the resized() function by writing the new size to the transcript and forwarding it to the client terminal.

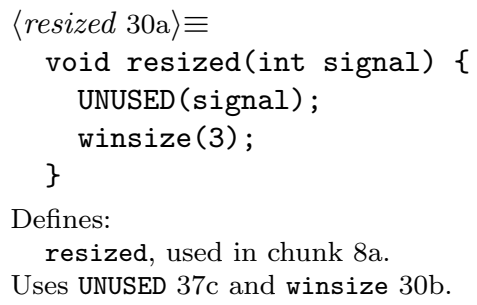

The actual reading and writing of the window size is done by winsize(), which takes a mode parameter. If the mode is 1 , the client applications terminal size will be set. If the mode is 2 , the terminal size will be written to the transcript. If the mode is 3 , both operations will be done, which is the usual case.

$30 \mathrm{~b}\langle$ winsize $30 \mathrm{~b} \equiv$

void winsize (unsigned int mode) \{

struct winsize size;

ioctl(STDIN_FILENO, TIOCGWINSZ, \&size);

if (mode \& 2)

if chunk11 (\&size) $<0)$

die ("writing window size", 0x11);

if ((mode \& 1) \&\& PTM)

Defines: 
winsize, used in chunks $18 \mathrm{c}$ 30a, 31b and 34c

Uses chunk11 18c die 23b and PTM 28c

Retrieving the window size requires ioctl.h for ioctl():

$\langle$ includes $8 \mathrm{~b}+\equiv$

\#include <sys/ioctl.h>

(15b) $428 \mathrm{f} 33 \mathrm{~b}>$

The client PTYs window size will be initialized now. This needs to take place before the client application is launched, because it probably requires an already configured terminal size when starting up. Writing the size to the transcript however would put the window size meta chunk before the start of session chunk, therefore winsize() s mode 1 is used.

$31 \mathrm{~b}\langle$ openpt $31 \mathrm{~b} \equiv$

winsize (1);

Uses winsize $30 \mathrm{~b}$

\section{A.10 Running the Target Application}

The doshell() function is run in the child process, whose only task is to set up all required PTY redirections and then execute the client command. Therefore, open file descriptors from the parent process which are no longer needed are closed early.

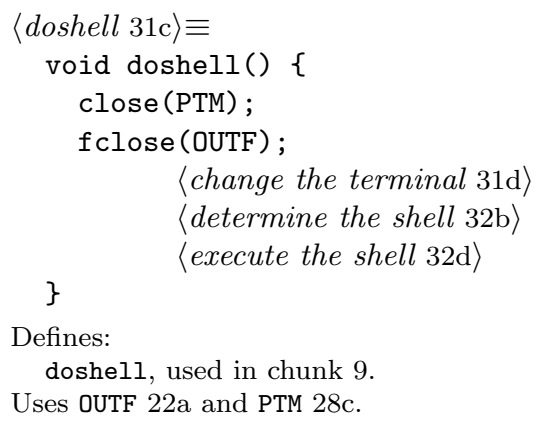

\section{Changing the Terminal}

Next, the child process changes its controlling terminal to be the PTY slave. In order to do that, it has to be placed in a separate session.

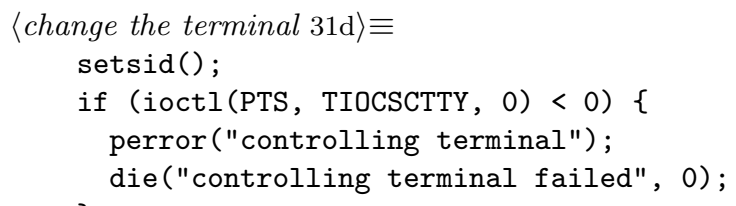


Standard input, output and error are bound to the PTY slave, which can then be closed.

32a <change the terminal $31 \mathrm{~d}+\equiv$

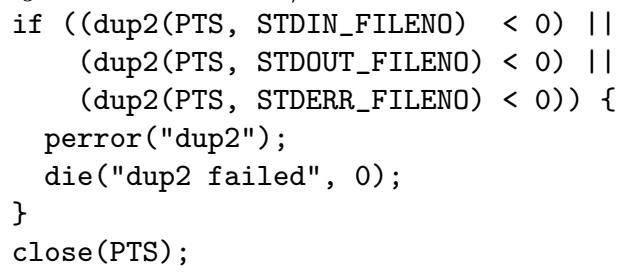

\section{Determining the Shell}

If the environment variable \$SHELL is set, its value is used. Otherwise the default is / $\mathrm{bin} / \mathrm{sh}$, which should exist on all Unix systems.

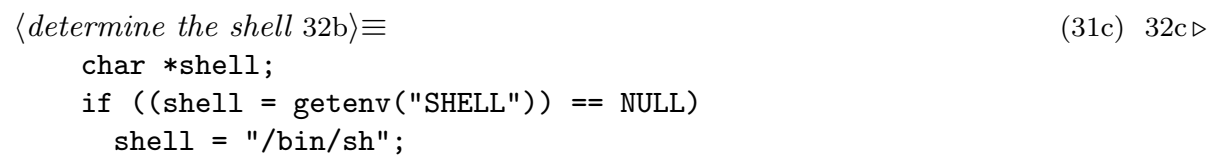

Next, the name of the shell, without any path components, is determined to be used as argument zero when executing the client command.

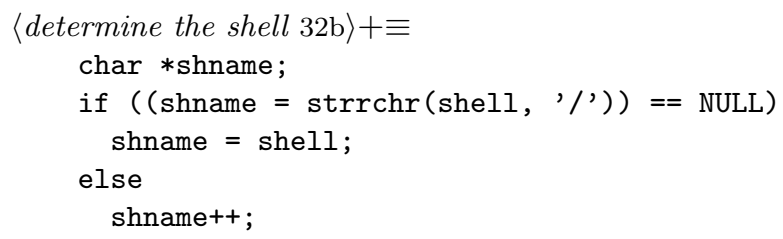

\section{Executing the Shell}

Finally, the execl () function is used to replace the currently running forscript process with the shell that has just been selected. If a target command has been specified using the -c option, it will be passed to the shell. Else, an interactive shell is launched using the $-i$ option.

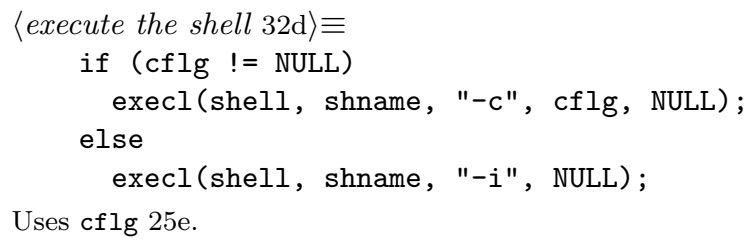


The forscript child process should now have been replaced with the shell. If execution reaches code after execl(), an error occured and the child process will terminate with an error message.

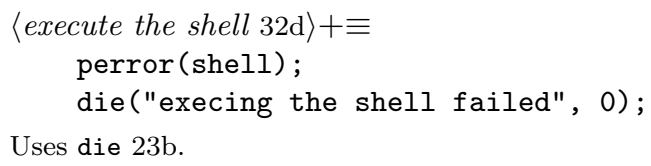

\section{A.11 Handling Input and Output}

While script forks twice and utilizes separate processes to handle input and output to and from the client application, forscript uses a single process for both tasks, taking advantage of the select() function (defined in select.h) that allows it to monitor several open file descriptors at once.

Input and output data will never be read simultaneously. Therefore, a single data buffer is sufficient. Its size is BUFSIZ bytes, which is a constant defined in stdio.h and contains a recommended buffer size, for example 8192 bytes. The number of bytes that have been read into the buffer by read() will be stored in count.

If the main loop exits, the child has terminated. done() is called to flush data and tidy up the environment.

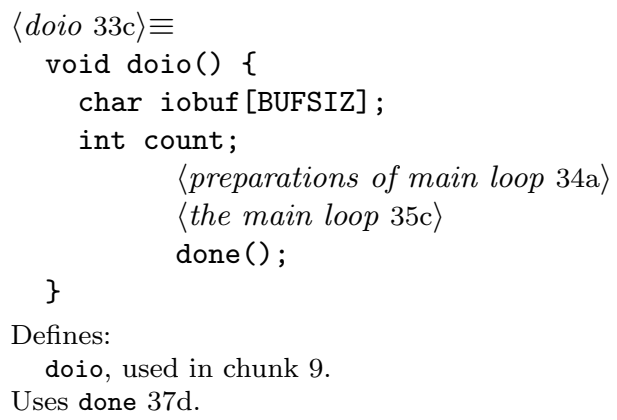

\section{Preparing the Main Loop}

The select () function is supplied with a set of file descriptors to watch, stored in the variable $f d s$. It returns in sr the number of file descriptors that are ready, or -1 if an error occured (for example, a signal like SIGWINCH was received). Additionally, it requires the number of the highest-numbered file descriptor plus one as its first parameter. On all Unix systems, stdin should be file descriptor 0 , 
but for maximum portability, forscript compares both descriptors and stores the value to pass to select() in the variable highest.

Uses PTM 28c

The variable drain determines whether the child has already terminated, but the buffers still have to be drained.

$34 \mathrm{~b}\langle$ preparations of main loop $34 \mathrm{a}+\equiv$

int drain $=0$;

33c $434 \mathrm{a} 34 \mathrm{c}$

Several metadata chunks need to be written. If the -a flag is not set, a file version chunk is written. Then begin of session, environment variables and locale settings. Finally winsize()s mode 2 is used to only write the window size to the transcript without sending a second SIGWINCH to the client.

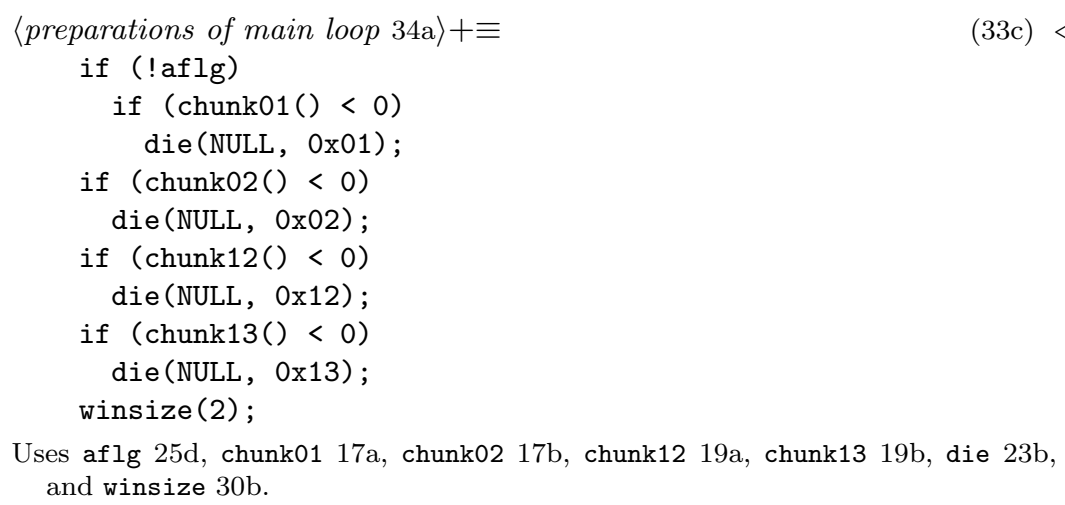

To be able to calculate the delay between I/O chunks, the monotonic clock available via clock_gettime() is used. The following code will initialize the timer:

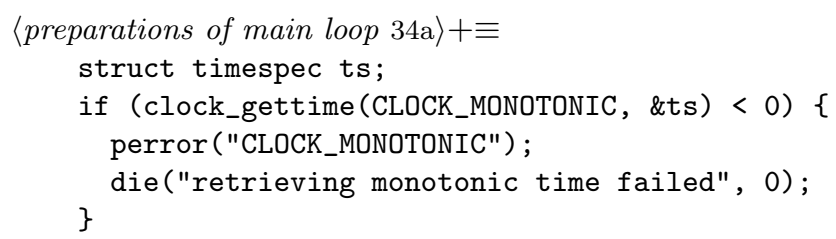

If the -q flag has not been supplied, forscript will display a startup message similar to scripts and write the same message to the transcript file. Note that this behavior differs from scripts: When called with -q, script would not output the startup message to the terminal, but record it to the typescript file nevertheless. This is required because scriptreplay assumes that the first line in the typescript is this startup message and will unconditionally suppress its 
output. forscript, however, has no such limitation and will not write the startup line to the transcript if the -q flag is set.

35a (preparations of main loop $34 \mathrm{a}+\equiv$ if (! qf lg) statusmsg(STARTMSG);

Uses STARTMSG $35 \mathrm{~b}$ and statusmsg $24 \mathrm{~b}$

$\langle$ constants $16 \mathrm{a}+\equiv$

const char * STARTMSG = "forscript started on $\%$, "

15b $416 \mathrm{~b} 38 \mathrm{c}>$ "file is $\% s \backslash r \backslash n "$;

Defines:

STARTMSG, used in chunk $35 \mathrm{a}$

\section{The Main Loop}

The main loop, which handles input and output, will run until the child process exits.

$35 \mathrm{c}\langle$ the main loop $35 \mathrm{c}$ 三 while ( CHILD >0) II drain) \{

\}

$\langle$ main loop body $35 \mathrm{~d}$,

Uses CHILD $8 \mathrm{~d}$

Since select() manipulates the value of $f d s$, it has to be initialized again in each iteration. First its value is cleared, then the file descriptors for standard input and the PTYs master are added to the set, then select() is called to wait until one of the file descriptors has data to read available. When in drain mode, select () may not be called to avoid blocking.

$\langle$ main loop body $35 \mathrm{~d}] \equiv$ if (!drain) \{

Uses PTM 28c

If the child process has terminated, there may still be data left in the buffers, therefore the terminals file descriptor is set to non-blocking mode. Reading will then continue until no more data can be retrieved. If drain mode is already active, this code will not be executed.

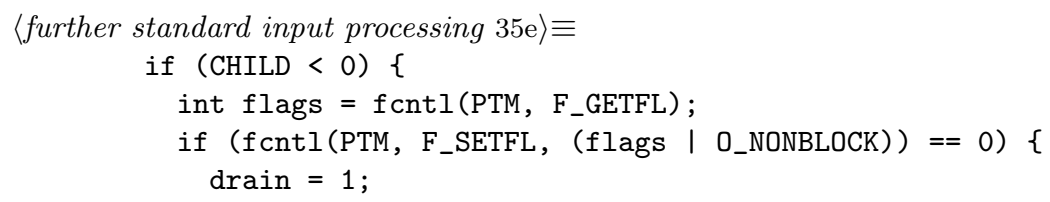




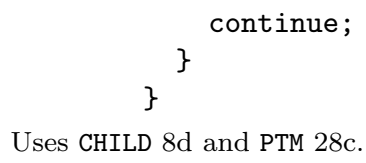

If select returns 0 or less, none of the file descriptors are ready for reading. This can for example happen if a signal was received and should be ignored. If the signal was SIGCHLD, notifying the parent thread of the childs termination, the signal handler will have set CHILD to -1 and the loop will finish after the buffers have been drained. If drain mode is already active, select() will not have been run, therefore this test is not needed then.

Execution does not reach this point if none of the file descriptors had data available. Thus it can be assumed that data will be written to the transcript file. Therefore chunk16() is called to calculate and write a delay meta chunk. After it has calculated the time delta, it will automatically update ts to contain the current time.

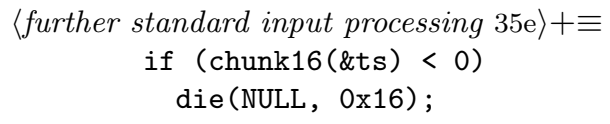

If user input is available, it will be read into the buffer. The data will then be written to the transcript file, having $\mathrm{SO}$ prepended and $\mathrm{SI}$ appended. Then it will be sent to the client application. When in drain mode, user input is irrelevant since the child has already terminated.

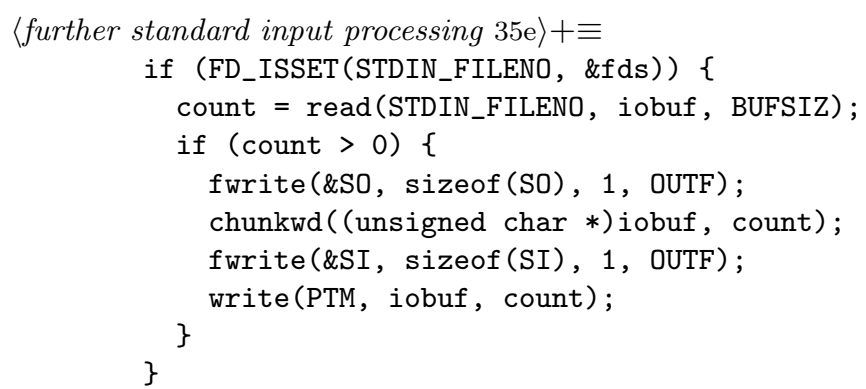

Regardless of whether in drain mode or not, if output from the client application is available, it will be read into the buffer and written to the transcript file and standard output. If there was no data to read, the buffer has been drained, drain mode ends and the main loop will terminate.

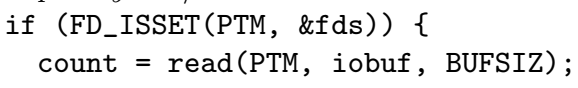




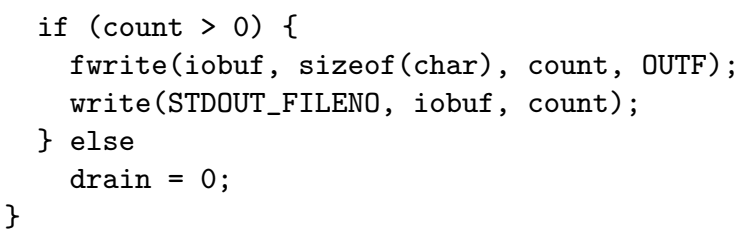

If the $-\mathrm{f}$ flag has been specified on the command line, the file should be flushed now that data has been written.

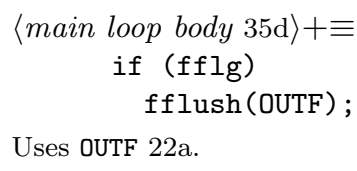

\section{A.12 Finishing Execution}

Since a signal handler can handle more than one signal, its number is passed as an argument. However, finish() only handles SIGCHLD, therefore it will ignore its argument. Its only task is setting CHILD to -1 , which will cause the main loop to exit as soon as possible.

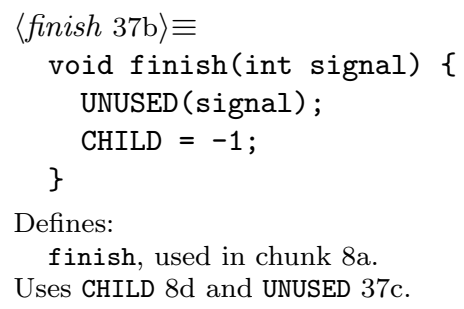

UNUSED is a macro that causes the compiler to stop warning about an unused parameter.

The function done() is called as soon as the main loop terminates. It cleans up the environment, resets the terminal and finishes execution. First, it has to fetch the exit status of the child process using wait().

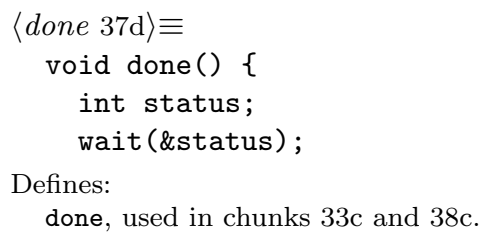


To be able to use wait(), wait.h must be included.

38a $\langle$ includes $8 \mathrm{~b}\rangle$

\#include <sys/wait.h>

(15b) $\triangleleft 33 \mathrm{~b}$

If the -q flag has not been supplied, forscript will write a shutdown message to both the terminal and the transcript file.

$38 \mathrm{~b}\langle$ done $37 \mathrm{~d}+\equiv$ if (!qflg)

statusmsg STOPMSG) ;

Uses statusmsg $24 \mathrm{~b}$ and STOPMSG $38 \mathrm{c}$

38c <constants $16 \mathrm{a}+\equiv$

const char * STOPMSG $=$ "forscript done on $\%$ s, "

15c $437 \mathrm{~d} 38 \mathrm{~d}$

"file is \%s\r\n";

Defines:

STOPMSG, used in chunk $38 \mathrm{~b}$

Uses done $37 \mathrm{~d}$

Finally, it will write an end of session chunk, close open file descriptors, reset the terminal and exit.

$\langle$ done $37 \mathrm{~d}+\equiv$

if (chunk03 (status) $<0$ )

die(NULL, 0x03);

fclose (DUTF) ;

close (PTM) ;

close (PTS);

if (tcsetattr(STDIN_FILENO, TCSADRAIN, \&TT) $<0)\{$ perror("tcsetattr on exit"); \}

Uses chunk03 18b die 23b OUTF 22a and PTM 28c 


\section{Chunk Index}

$\langle$ change the terminal $31 \mathrm{~d}$,

$\langle$ chunks 17a

$\langle$ chunkw 21〉

$\langle$ chunkwhf 22d

$\langle$ chunkwm 23a

$\langle$ constants 16a

$\langle$ declarations and definitions $15 \mathrm{~b}$

$\langle$ determine the shell $32 \mathrm{~b}$.

$\langle$ die 23b

$\langle$ doio 33c

Ldone 37d

$\langle$ doshell $31 \mathrm{c}$

$\langle$ execute the shell $32 \mathrm{~d}$.

$\langle$ featuretest $27 \mathrm{~b}$

$\langle$ finish 37b

$\langle$ fork subprocesses $8 \mathrm{c}$

$\langle$ forscript.c $7 \mathrm{a}$

$\langle$ functions $15 \mathrm{c}$

$\langle$ further standard input processing 35e

$\langle$ globals 8d

$\langle$ includes $8 \mathrm{~b}$

$\langle$ macros $37 \mathrm{c}\rangle$

$\langle$ main $7 \mathrm{~b}|$

$\langle$ main loop body $35 \mathrm{~d}$.

〈open new pseudo terminal 28e

〈open output file 26c

Lopenpt 31b

$\langle$ preparations of main loop 34a

$\langle$ process command line options 25b

$\langle$ register signal handlers $8 \mathrm{a}$

$\langle$ resized 30a

set my name 24c

$\langle$ statusmsg $24 \mathrm{~b}$.

$\langle$ swrite $22 \mathrm{~b}$ <

the main loop $35 \mathrm{c}$

$\langle$ winsize $30 \mathrm{~b}$ 


\section{Identifier Index}

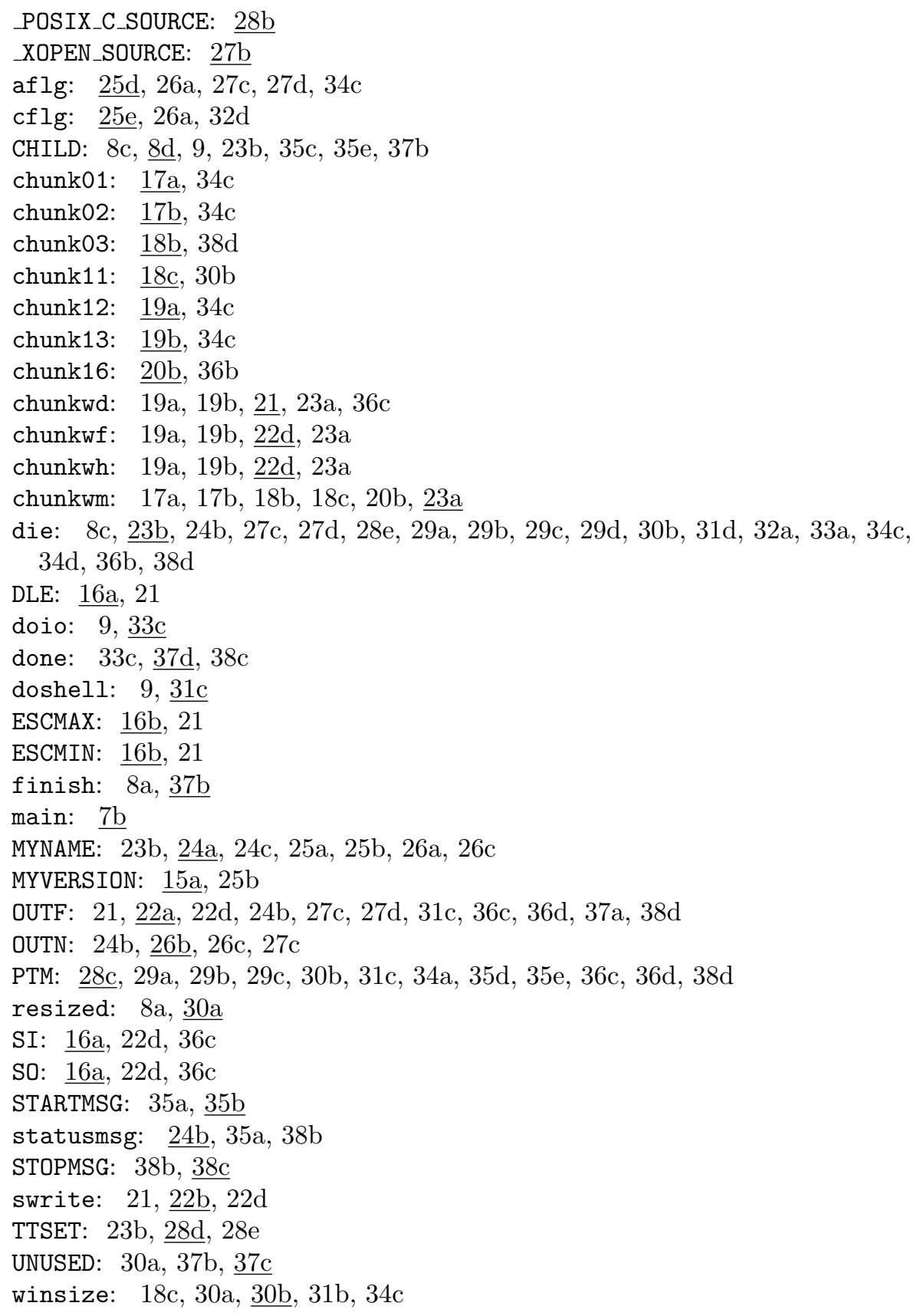

